\title{
Entscheidungen, Entscheidungsfindung und Entscheidungsunterstützung in der Immobilienwirtschaft: Eine systematische Literaturübersicht
}

\author{
Patrick Krieger (D) C Carsten Lausberg
}

Eingegangen: 4. März 2018 / Überarbeitet: 12. Januar 2020 / Angenommen: 22. Januar 2020 / Online publiziert: 7. Februar 2020

(C) Der/die Autor(en) 2020

Zusammenfassung Dieser Artikel untersucht den Stand der immobilienwirtschaftlichen Forschung zu menschlichen Entscheidungen und Entscheidungsunterstützungssystemen (EUS). Er baut auf einer systematischen Literaturrecherche auf, die die Entwicklung der Forschung, untersuchte immobilienwirtschaftliche Felder, angewendete Methoden, Verbindungen zu anderen Disziplinen sowie favorisierte Zeitschriften identifiziert. Es werden Problemstrukturen aufgezeigt, die auf drei Bereiche verdichtet werden können: (1) Die primäre Forschungsleistung fließt in die Bereiche Investitionen und Bewertung. Andere immobilienwirtschaftliche Disziplinen sind bisher unterrepräsentiert. (2) Ein Wissenstransfer zwischen der verhaltensorientierten Immobilienforschung und der Informatik ist kaum zu beobachten. Insgesamt scheint die Forschung über immobilienwirtschaftliche Entscheidungsunterstützungssysteme noch am Anfang zu stehen. (3) Der in der Immobilienwirtschaft vorherrschende Risikobegriff ist unzureichend und muss um eine psychologische Dimension erweitert werden, um sein Potential für immobilienwirtschaftliche EUSAnwendungen entfalten zu können.

Schlüsselwörter Entscheidung · Entscheidungsfindung ·

Entscheidungsunterstützung $\cdot$ Verhaltensorientierte Ökonomie $\cdot$ Psychologie der Entscheidung

\footnotetext{
P. Krieger $(\bowtie)$

Technische Universität Berlin, Kaiser-Friedrich-Straße 39, 10117 Berlin, Deutschland

E-Mail: patrick.krieger@campus.tu-berlin.de

C. Lausberg

Immobilienwirtschaftliches Institut für IT, Hochschule für Wirtschaft und Umwelt

Nürtingen-Geislingen, Parkstraße 4, 73312 Geislingen/Steige, Deutschland
} 


\title{
Decisions, decision-making and decisions support systems in real estate: a systematic literature review
}

\begin{abstract}
This article examines the state of real estate research on human decisions and decision support systems (DSS). It builds upon a systematic literature review, which analyses real estate research fields, used methodologies, connections to other disciplines, and favored journals for publications. Problem structures are revealed which point to three areas that require further research: (1) Research focusses on real estate investment and valuation. Other fields are under-researched. (2) There is almost no knowledge transfer between behavioral real estate research and computer science. Overall, real estate research on DSS is only at its beginning. (3) The predominant definition of risk in real estate is insufficient and needs to be augmented by a psychological dimension to unfold its potential for DSS applications in real estate.
\end{abstract}

Keywords Decision-Making · Decisions · Decision Support · Behavioral Economics $\cdot$ Decision Biases $\cdot$ Decision Aids

\section{Einleitung}

Entscheidungen und ihre Konsequenzen sind seit jeher Gegenstand der Wirtschaftswissenschaften. Doch erst mit der Öffnung für die Verhaltenswissenschaften und andere Disziplinen sind Entscheidungen in den Mittelpunkt der Ökonomie gerückt. Durch die Abkehr vom Homo oeconomicus und die Einbeziehung von Erkenntnissen aus Psychologie, Soziologie, Neurologie und anderen Disziplinen kann die Ökonomie besser erklären, wie sich Menschen verhalten und besser gestalten, wie sich Menschen verhalten sollten. Dies ist zumindest der Anspruch der entscheidungsorientierten Betriebswirtschaftslehre, die im deutschen Sprachraum auf Heinen (1969) zurückgeht und insbesondere in der Managementlehre bis heute eine hohe Bedeutung hat. In der Immobilien-BWL ist dieser Ansatz anscheinend nicht sehr verbreitet, wie als Beiprodukt dieser Arbeit herauskam. Es gibt zwar immer wieder Autoren, die sich auf ihn berufen, doch nur wenige beschäftigen sich explizit mit Entscheidungen. Über die Gründe können wir an dieser Stelle nur Vermutungen anstellen: Möglicherweise ist die Dominanz derjenigen zu groß, die die Betriebswirtschaftslehre als exakte Wissenschaft ansehen und dies als Widerspruch zu einer psychologischen Perspektive betrachten; eventuell sind andere Themen in den Augen der meisten Forscher dringender und interessanter; oder vielleicht sind die Barrieren zu hoch, um sich interdisziplinär mit der menschlichen Entscheidungsfindung zu befassen. Jedenfalls ist es das Ziel dieses Artikels, den bisherigen Wissensstand über Entscheidungen, Entscheidungsfindung und Entscheidungsunterstützung im Zusammenhang mit Immobilien zusammenzutragen.

Im Folgenden definieren wir die Entscheidungsfindung (synonym: das Entscheiden) als den Entscheidungsprozess, der die Problemidentifikation, die Auswahl der Kriterien, die Erarbeitung der Lösungsalternativen, die Identifizierung der besten Alternative anhand der spezifizierten Kriterien, die Planung der Umsetzung sowie 
die Überprüfung des Ergebnisses umfasst (siehe z.B. Guo 2008). Die Entscheidung wird hierbei als die endgültig umgesetzte Alternative verstanden, wobei zwei Problemfelder vorhanden sind:

1. dass die umgesetzte Alternative von der eigentlich beabsichtigten Alternative abweicht oder

2. dass eine Alternative ausgewählt wird, die ungeeignet für das angestrebte bzw. erwartete Ergebnis ist.

Ersteres ist von untergeordneter Bedeutung, da im Gesamtprozess die umgesetzte Entscheidung und das dadurch erzielte Ergebnis relevant sind. Letzteres ist wichtiger, da das Wissen über die Gründe (z. B. sog. Cognitive Biases) und eventuelle Interventionsstrategien (sog. Debiasing) zur Verbesserung der Entscheidungsergebnisse beitragen können. Zur Unterstützung der menschlichen Entscheidungsfindung tragen viele Maßnahmen bei - von einfachen Ablaufdiagrammen bis zu komplexen Prozessmodellen, von Checklisten bis zu Instrumenten mit künstlicher Intelligenz. Power et al. (2011) definieren die hierfür entwickelten Systeme,

[...] [as a] a class of computerized information systems that support decisionmaking activities [...] which enhance users' effectiveness in making complex decisions [...].

Diese Definition umfasst den gesamten Entscheidungsprozess und erweitert die Problemstellung um den Begriff der Effektivität. Im Folgenden wird darunter verstanden, dass das erzielte Ergebnis durch die Entscheidung mit EUS im Durchschnitt zumindest gleich oder oberhalb der Entscheidungsergebnisse von Personen ohne EUS liegen sollte. Hierbei wird vorausgesetzt, dass die Entscheidungsergebnisse in eine entsprechende Über- und Unterordnung gebracht werden können, was im weiteren Verlauf auch als „User Effectiveness“ oder Entscheidungsperformance bezeichnet wird.

Das hierdurch umrissene Forschungsgebiet kann auf drei einflussreiche Artikel amerikanischer Wissenschaftler zurückgeführt werden: Ende der 1980er Jahre stießen Gregory B. Northcraft und Margaret A. Neale grundsätzliche Überlegungen zum immobilienwirtschaftlichen Entscheiden an, begann Julian Diaz III. eine Forschungsserie dazu, wie Entscheidungen bei Immobilienbewertungen getroffen werden und veröffentlichte Robert R. Trippi einen Überblick über computergestützte Systeme zur Unterstützung von Immobilienentscheidungen (Northcraft und Neale 1987; Trippi 1989; Diaz III. 1990). Daher wird eine Bestandsaufnahme der einschlägigen Literatur ab 1986 unternommen, die das bisher Erreichte zusammenfasst und auf bestehende Forschungslücken hinweist.

Die Untersuchung baut auf einer vereinfachten Variante ${ }^{1}$ einer systematischen Literaturrecherche auf. Das nächste Kapitel beschreibt die Suchstrategie, die Datenerhebung sowie die Stichprobe. Das dritte Kapitel enthält eine Einführung in

\footnotetext{
1 Die systematische Literaturanalyse gliedert sich an die Meta-Analyse an, um Effektgrößen einer Untersuchung über mehrere Studien zu plausibilisieren. Da es nicht das Ziel des Artikels ist Effektgrößen zu ermitteln/plausibilisieren, wurde die detaillierte Artikelanalyse gegen eine Analyse deskriptiver Merkmale ersetzt.
} 
Abb. 1 Schematischer Aufbau zu den erwarteten Transmissionskanälen der Forschungsgebiete

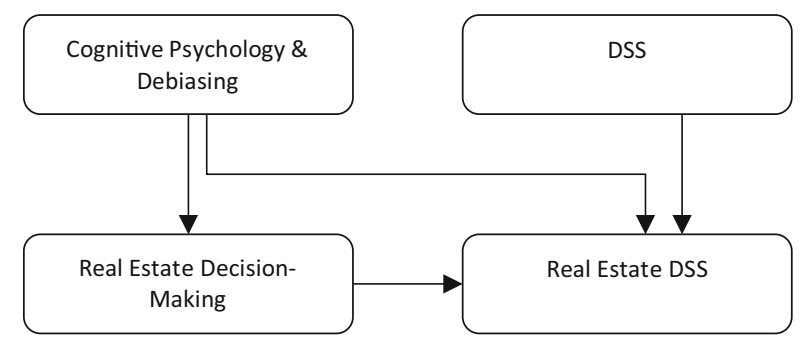

die allgemeine verhaltensorientierte Betriebs- und Finanzwirtschaftslehre, die zur neoklassischen Lehre abgegrenzt wird. Das vierte Kapitel vergleicht die Entwicklung der immobilienwirtschaftlichen und der allgemeinen betriebswirtschaftlichen Forschung. Das fünfte Kapitel gibt einen Überblick über die Forschung zu EUS. Abschließend werden die Ergebnisse der systematischen Literaturrecherche rekapituliert und Forschungslücken besprochen.

\section{Erläuterungen zur Methodik}

Die vorliegende Literaturrecherche orientiert sich an der Methodik von Arnott und Pervan (2005) zur kritischen Analyse der EUS-Forschung. Hierbei handelt es sich um eine vereinfachte systematische Literaturanalyse relevanter Artikel in internationalen wissenschaftlichen Zeitschriften. Die Untersuchung folgt den Prinzipien systematischer Literaturanalysen, ohne den Anspruch einer Meta-Analyse zu erheben. Die erhobene Stichprobe wird in die Teile „Real Estate Decision-Making“ und „Real Estate DSS“ geordnet. Diese beiden resultierenden Spezialfelder werden dann mit den jeweils übergeordneten Forschungsfeldern „Cognitive Psychology \& Debiasing“ bzw. „DSS \& Debiasing“ verglichen. Der Vergleich soll eine Einschätzung zum Entwicklungsstand des jeweiligen Spezialgebiets liefern und eventuelle Forschungslücken und weitere Auffälligkeiten aufdecken. Des Weiteren wird der Wissenstransfer zwischen den jeweiligen vorgenannten Bereichen betrachtet. Die Abb. 1 fasst den schematischen Aufbau zusammen.

\subsection{Recherche}

Die Recherche erfolgte in einem dreistufigen Prozess. In der ersten Stufe wurde eine allgemeine Literaturrecherche ${ }^{2} \mathrm{zu}$ den Themenfeldern „EUS“ und „Behavioral Economics“ durchgeführt, um die Ergebnisse der Stichprobe in den allgemeinen Kontext der verhaltensorientierten Forschung und der Forschung zu Entscheidungsunterstützungssystemen einordnen zu können. In der zweiten Stufe schloss sich eine systematische Recherche zur Erhebung einer Stichprobe an. Für diese Stichprobe wurden nur publizierte Artikel in wissenschaftlichen Zeitschriften einbezogen.

\footnotetext{
2 Die allgemeine Literaturrecherche wurde ohne die Aufstellung strikter Suchparameter und Quellentypen durchgeführt, da sie nicht quantitativ analysiert wurde.
} 
Das Hauptkriterium war die Verfügbarkeit in englischer Sprache, da in dieser Form zweifellos der größte Teil der Forschungsergebnisse vorliegt. Zur Recherche wurden die internationalen Datenbanken Taylor \& Francis, WileyOnline, ScienceDirect, EmeraldInsight, SpringerLink sowie GoogleScholar verwendet, die den Großteil der wissenschaftlichen Immobilien-Journale abdecken, d.h. solcher Zeitschriften, die eine Begutachtung durch Experten vorsehen, meistens im Doppelblindverfahren (Double-blind Peer Review). Die Artikel wurden mittels einer Kombination aus den Suchbegriffen Decision, Support, Expert, System, Real Estate, Process, Behavioral, Bias und Heuristics ermittelt. Danach wurde überprüft, ob die jeweils ermittelte Artikelüberschrift zum Themenbereich passt und ob der Artikel einer genaueren Prüfung unterzogen wird (Punkt „Artikel nach Titelanalyse“). ${ }^{3}$ In der dritten Stufe wurden zusätzlich auch die Literaturverzeichnisse der berücksichtigten Artikel nach weiteren relevanten Quellen durchsucht. ${ }^{4}$ Nach einer genaueren Prüfung des Artikels wurde entschieden, ob der Artikel in die Stichprobe aufgenommen oder von der Stichprobe ausgeschlossen wird. Maßgeblich hierfür sind der Immobilienbezug, die Entscheidungsfindung (im psychologischen Sinne) sowie Entscheidungsunterstützungssysteme. Bei der Suche mit den Begriffen „Decision Real Estate“ wurde z. B. ein Artikel mit dem Titel ,Implications of valuation methods for the management of property assets" bei der Titelanalyse in die Stichprobe aufgenommen. Dieser Artikel genügte jedoch nach detaillierter Prüfung nicht den angegebenen Stichprobenkriterien, da der psychologische Entscheidungsprozess nicht Teil des Artikels war. Des Weiteren schloss sich eine systematische Literaturrecherche zur deutschsprachigen Literatur an. Die Funde wurden jedoch nicht in die Stichprobe einbezogen, sondern nur an geeigneten Stellen im Text ergänzend erwähnt.

\subsection{Stichprobe}

Aus Kombinationen der vorgenannten Suchbegriffe wurden insgesamt 457 Artikel auf Basis der Artikeltitel vorselektiert. Nach Prüfung des Abstracts wurden insgesamt 354 Artikel aussortiert, die nicht zum Thema „Entscheiden, Entscheidungen und Entscheidungsunterstützung“ passen. Insgesamt wurden 133 relevante Artikel gefunden, wobei insgesamt 7 weitere Artikel durch die Suche innerhalb der jeweiligen Literaturverzeichnisse identifiziert wurden. Da für die Stichprobe nur englischsprachige doppelt blind begutachtete Artikel berücksichtigt werden, konnten insgesamt 15 Artikel innerhalb der 103 Artikel identifiziert werden, die diesen Kriterien nicht genügen. Die finale Stichprobe umfasst insgesamt 88 Artikel, von denen 48 Artikel zum Thema „Real Estate Decision-Making“ und 40 zum Thema „Real Estate DSS“ gehören. Die 88 Artikel verteilen sich auf insgesamt 18 Journale zu den unterschiedlichen Themenbereichen der Immobilienwirtschaft. Die Abb. 2 veranschaulicht den Selektionsprozess.

\footnotetext{
3 Entsprechende Doubletten wurden beim Import durch die Literatur-Software bereits angefangen.

${ }^{4}$ Da die Plattformen eine sehr große Menge an Artikeln umfassen, besteht die Gefahr, dass relevante Artikel nicht identifiziert wurden. Mit der Analyse der Literaturverzeichnisse wird versucht, eine möglichst vollständige Stichprobe sicherzustellen.
} 
Abb. 2 PRISMA Diagramm zur resultierenden Stichprobe. Während der allgemeinen Recherche haben wir insgesamt 38 deutsche Artikel und Monographien identifiziert, wobei hiervon 4 Publikationen zitiert wurden. Die deutschen Artikel sind nicht in der Abbildung enthalten (RE DM Real Estate DecisionMaking, RE DSS Real Estate Decision Support Systems)

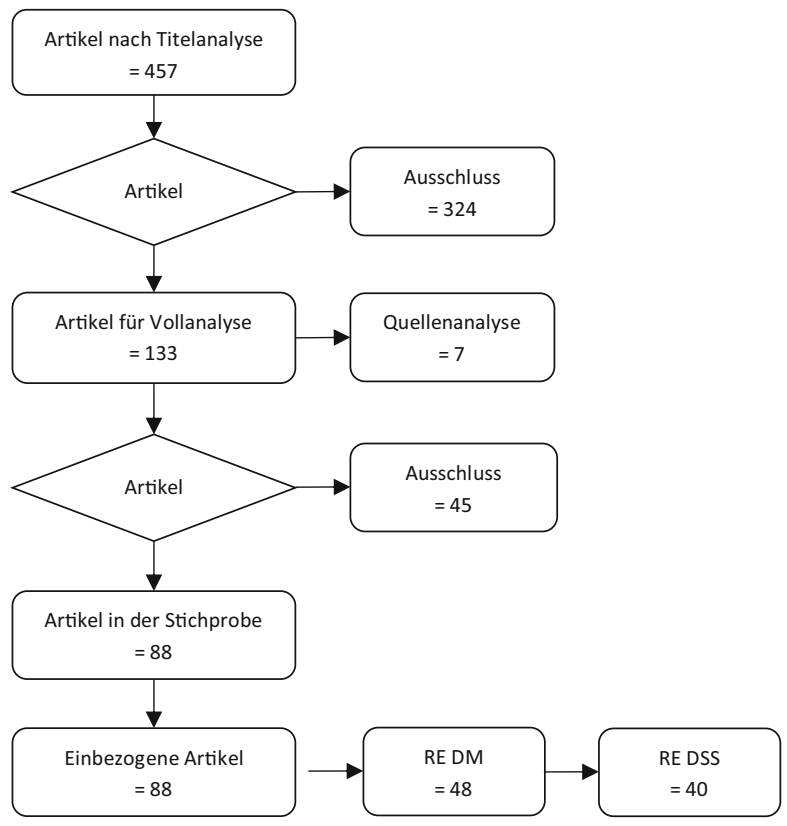

\subsection{Datenerhebung}

In einem nachgelagerten Schritt wurden für die folgenden Merkmale Anzahl der Artikel, Anzahl der Forscher, Internationalität, und Praxisbeteiligung festgelegt. Für die spezielle Auswertung wurden die Merkmale Disziplin der Immobilienwirtschaft, Untersuchungsgegenstand, Methodik und Ergebnisse festgelegt. Abschließend wurden alle Merkmale für die ausgewählten Artikel erhoben und tabellarisch erfasst.

\section{Einführung in die Forschung der verhaltensorientieren Ökonomie}

Die Verhaltensökonomik entstand Anfang der 1970er-Jahre, als sich Verhaltenswissenschaftler zunehmend mit wirtschaftlichen Entscheidungen von Individuen und Organisationen beschäftigten. Insbesondere legten sie den Fokus auf die Abweichungen zwischen dem Homo oeconomicus und den menschlichen Verhaltensweisen in der Wirklichkeit. Diese Entwicklung ist insofern bemerkenswert, da es schon in der Neoklassik und anderen ökonomischen Denkrichtungen intensive Auseinandersetzungen mit dem menschlichen Verhalten gab und Marshall (1920) feststellte:

[...] [that] [e]conomics is a study of mankind in the ordinary business of life;

[...] Thus it is on the one side a study of wealth; and on the other, and more important side, a part of the study of man.

Dennoch zieht sich die Annahme des Homo oeconomicus durch die einflussreichen Theorien, die auf den objektiven Optima der Erwartungsnutzentheorie auf- 
bauen. Nur entspricht die Annahme des vollständig rational handelnden Menschen kaum dem tatsächlichen Handeln. Simon (1955) stellte hierzu fest:

[that] the distance is so great between our present psychological knowledge of the learning and choice processes and [...] economic and administrative theory $[\ldots]$.

Beide Forschungsrichtungen brachten in den letzten Jahrzehnten gegensätzliche Forschungsergebnisse hervor, und es bleibt eine Diskussion, ob sich die von der Psychologie aufgedeckten (Verhaltens-)Anomalien im Markt ausgleichen oder bestehen bleiben.

Nach der neoklassischen Entscheidungstheorie kann jede unsichere Entscheidung anhand einer Nutzen-Risiko-Relation bewertet werden, woraus eine dominante Variante aus einer endlichen Anzahl an Alternativen resultiert (von Neumann und Morgenstern 1947). Im Kontext der Portfoliotheorie ergibt sich die Entscheidung für eine Mischung von Anlagealternativen rechnerisch aus den Größen Rendite, Volatilität, Kovarianz und Nutzen (Markowitz 1952). Diese Modelle sind unbestritten ein Meilenstein in der ökonomischen Theorie, aber selbst nach Anpassungen nur eingeschränkt auf Immobilien anwendbar, u. a. weil die Datensituation fundamental anders ist als bei börsennotierten Wertpapieren (Viezer 2010). Ähnlich verhält es sich mit Modellen zur Messung von Immobilienrisiken: Auf der einen Seite müssen die Maßsysteme logisch widerspruchsfrei sein (Rockafellar et al. 2006; Artzner et al. 1999; Pedersen und Satchell 1998). Auf der anderen Seite müssen die Maße flexibel genug sein, um die empirischen Daten vernünftig abbilden zu können. Letzteres stellt immer noch ein großes Problem dar, weil viele von der neoklassischen Theorie vorgeschlagene quantitative Risikomaße Immobilienrisiken nicht richtig ausdrücken können. Die Auswahl aus einer endlichen Menge an Alternativen kann auch auf subjektivem Wege geschehen. Ein Verfahren für diese Aufgabe ist der Analytische Hierarchieprozess (AHP), der von Saaty (1987) entwickelt wurde. Dieser Ansatz baut auf einem normativen Entscheidungsprozess auf, der die Phasen Informationsgewinnung, Bewertung, Auswahl und Nachschau umfasst (Simon 1977). Das von Saaty entwickelte Sortierverfahren soll dafür sorgen, dass die Entscheidungsaxiome für widerspruchsfreie Entscheidungen erfüllt werden (Savage 1972). Insgesamt handelt es sich um ein subjektiv-qualitatives Verfahren, das - wie ähnliche Verfahren, die als Nutzwertanalyse oder Scoring bekannt sind - in der Wirtschaft großen Anklang gefunden hat (VÖB-Kommission für Bewertungsfragen 2006; Adair und Hutchison 2005). Dass Individuen von normativen Prozessen abweichen, ist nicht verwunderlich, wohl aber, dass sie davon abweichen, obwohl sie die Norm kennen und damit angeblich einen höheren Nutzen verbinden.

In vielen Fällen sind Individuen nicht bereit hohe kognitive Anstrengungen zu unternehmen und geben sich auch mit einer suboptimalen Lösung zufrieden. Simon (1955) erahnte diese Kluft zwischen menschlichen Entscheidungen und mathematischen Modellen:

[...] [A]ctual human rationality-striving can at best be an extremely crude and simplified approximation to the kind of global rationality that is implied, for example, by game-theoretical models. (Simon 1955, S. 101) 
Jedoch ist dies keine Erklärung dafür, warum die Axiome für rationale Entscheidungen verletzt werden (Kahneman und Tversky 1979). Des Weiteren stellt sich die Frage, ob das normative Entscheidungsverhalten auch in einem ,ineffizienten“ System wirklich zu besseren Ergebnissen führt. Der menschliche Entscheidungsprozess wird maßgeblich vom Individuum in Verbindung mit seinem sozialen Umfeld geprägt. ${ }^{5}$ Dies beeinflusst nicht nur die Entscheidung, sondern auch die Akzeptanz gegenüber Systemen, die bei Entscheidungen unterstützen sollen und worauf im weiteren Verlauf des Artikels noch eingegangen wird.

Ähnliches gilt auch für die Risiko-Rendite-Funktion ${ }^{6}$, denn der Risikobegriff ist gleich in mehrerlei Hinsicht problematisch. Die Diskussion über Auswirkungen von Spitzentechnologie (z. B. potentielle Risiken durch Hochspannungsmasten) zwischen Laien und Experten zeigt, dass ein quantitatives Risikomaß nur Teile des Risikos abbilden kann (z. B. Luhmann 1997). Dem Risikobegriff liegt eine soziale Dimension inne (Slovic 1999), die von Person zu Person (auch rational) sehr unterschiedlich wahrgenommen werden kann (Hornig 1993). Beispielsweise wird es als Unterschied empfunden, ob eine Person durch einen Dritten einem Risiko ausgesetzt wird oder sich die Person selbst diesem Risiko aussetzt. Die Eintrittswahrscheinlichkeit des Risikos verändert dies nicht, sondern es ändert sich lediglich die Selbstbestimmtheit des Betroffenen, eben die soziale Interaktion als eine zusätzliche Dimension des Risikos. Trotz quantitativer Risikomaße, die zu guten Entscheidungen führen können, werden Abweichungen von der „rational/mathematischen“ Norm festgestellt, die beispielsweise mittels eines Risiko-Rendite-Funktionales nicht zu erklären sind (de Bondt und Thaler 1985).

Dieses Phänomen war Gegenstand einer breit angelegten Forschungsagenda der Psychologen Tversky und Kahneman (1974). Sie untersuchten unter anderem die Entscheidungen von Experten, die mit Statistik vertraut sind. Sie zeigten, dass das Entscheidungsverhalten dieser Probanden trotz ihres Wissens systematisch vom rationalen Verhalten abweicht. Ihr Erklärungsansatz, der später von anderen Wissenschaftlern aufgenommen wurde, ist die Unterscheidung in zwei Systeme menschlicher Kognition. Zur Organisation dieser widersprüchlichen Ergebnisse wird oftmals die Unterscheidung zwischen Intuition und Denken angeführt. Die Arbeiten von Stanovich und West (2000) sowie Sloman (1996) unterscheiden in zwei Systeme menschlicher Kognition. System 1 ist für die schnelle Verarbeitung von Prozessen vorgesehen, die die Umwelt mittels Heuristiken abstrahiert. Diese Heuristiken sind in vielen Fällen gut, aber führen in einigen Fällen zu fehlerhaften Entscheidungen. Das System 2 wird mit einer langsamen Verarbeitung von Informationen in Verbindung gebracht, die die Aufmerksamkeit des Individuums fordert. Diese Art der Kognition ist flexibel und gewissenhaft, aber anstrengend. Wenn ein Individuum einen Reiz wahrnimmt, werden diese Informationen erst durch das System 1 verarbeitet und bei Bedarf an das System 2 weitergereicht (de Neys 2006).

\footnotetext{
5 Das Spektrum der Entscheidungsfindung erstreckt sich z. B. über inkrementelles (Lindblom 1959), organisationsprozedurales (Das und Teng 1999; Huber 1981), politisches (Pfeffer 1981), naturalistisches (Klein 1998) und Papierkorb-Entscheiden (Cohen et al. 1972).

${ }^{6}$ Hiermit ist der funktionale Zusammenhang zwischen Rendite und Risiko beim effizienten Rand und der Kapitalmarktlinie gemeint.
} 
Die bekanntesten Heuristiken sind die Verfügbarkeits- und Repräsentativitätsheuristik sowie die Anpassungs- und Ankerheuristik (Kahneman 2003). Die Verfügbarkeitsheuristik beschreibt die menschliche Einschätzung von Wahrscheinlichkeiten anhand im Gedächtnis verfügbarer und vergleichbarer Situationen (Schwarz et al. 1991; Tversky und Kahneman 1973). Dies ist insofern problematisch, da die erinnerte Stichprobe nicht mit der Grundgesamtheit übereinstimmen muss und somit falsche Einschätzungen folgen können. Des Weiteren neigen Individuen auch dazu, die Wahrscheinlichkeit von Ereignissen anhand von Stereotypen zu bewerten, die weder mit der Realität übereinstimmen müssen noch verlässlich sind (Gigerenzer und Hoffrage 1995; Tversky und Kahneman 1973). Die aufgeführten Heuristiken können ein oder mehrere Rationalitätsaxiome oder z. B. die Bayes-Statistik verletzen, die für ein rationales Entscheiden zwingend notwendig wären. Beispielsweise wird unterbewusst die Anker- und Anpassungsheuristik aktiviert, die als Referenzwert zur Beurteilung von Alternativen in einer Entscheidungssituation dient, aber das Transitivitätsaxiom verletzen kann (Brewer und Chapman 2002; Strack und Mussweiler 1997; Tversky und Kahneman 1974). ${ }^{7}$

Parallel zur Erforschung von Heuristiken und Biases wurden auch die Lernfähigkeiten von Individuen in Entscheidungssituationen untersucht. Gigerenzer und Kurzenhäuser (2005) gehen davon aus, dass Individuen in der Lage sind „smarte“ Heuristiken auszubilden, die ebenso präzise wie statistische Modelle sein können. Daher stellt sich die Frage, welche Informationen bzw. welches Feedback benötigt werden, um solche Heuristiken zu erlernen. Dieses sogenannte „Feedback-Learning “ wird in eine Querschnitts- und eine Zeitreihenanalyse unterteilt. Erstere umfasst multikriterielle Auswahlentscheidungen unter Unsicherheit (Multiple-Cue Probability Learning, MCPL) und letztere subjektive Schätzungen (Judgmental Forecasting) (Karelaia und Hogarth 2008; Lawrence et al. 2006; zur Übersicht). Die Probanden werden bei diesen Experimenten aufgefordert relevante Einflüsse zu identifizieren und zu gewichten, um ein Objekt oder eine zeitliche Entwicklung einzuschätzen. Diese Aufgabenstellung können Individuen gut erlernen, wenn gewisse Voraussetzungen vorhanden sind. Eine wesentliche Voraussetzung ist der Realitätsbezug der Aufgabe. Je näher die Aufgabe an der Realität des Individuums ist, desto höher ist die Aussagekraft der Testergebnisse (Brunswik 1955). Des Weiteren müssen genug Wiederholungen in Verbindung mit nützlichen Feedback-Informationen zur Verfügung stehen (Lagnado et al. 2006). Durch das Präsentieren von geeigneten Feedback-Informationen können die Probanden ihre Einschätzungen anpassen und bessere Schätzergebnisse abgeben, was schlussendlich zu einer erlernten Heuristik führen soll. Allerdings kommt es stark auf die Art der Feedback-Information an. Performance Feedback, also die Abweichungen von Einschätzung und eingetretenem Ergebnis, ist in diesem Zusammenhang weitaus ineffektiver als kognitives Feedback, das Auskunft über die Aufgabenstruktur gibt (Sengupta 1995; Balzer et al. 1994). Wenn unterschiedliche Faktoren für eine Einschätzung benötigt werden, können Individuen diese Faktoren auch entsprechend gewichten, selbst wenn

\footnotetext{
7 Eine umfangreiche Aufstellung von Biases ist in Carter et al. (2007) und Arnott (2006) verfügbar. Siehe für eine deutsche Sichtweise auch Mahlendorf (2008), der psychologische Fallstricke für Investitionsentscheidungen beleuchtet.
} 
die Zuverlässigkeit der Faktoren variiert (York et al. 1987). Allerdings sinkt die Performance von Probanden, wenn eine Problemstellung viele Faktoren zur Auswahl umfasst. Hier können statistisch kalibrierte Modelle die Leistung erhöhen (Grove et al. 2000; Dawes et al. 1989). Diese Herangehensweise liegt bereits nahe an einem computergestützten Entscheidungsunterstützungssystem und zeigt eine Möglichkeit zur Integration psychologischer Erkenntnisse.

In Ergänzung zu diesen Erkenntnissen stehen die Ergebnisse zu subjektiven Prognosen. Früher galten statistische Prognosen als bestmögliche Schätzung (siehe bspw. Meehl 1954, 1965). Mathews und Diamantopoulos (1986), Brown (1996, 1997) sowie Lawrence und O'Connor (1996) zeigen, dass die subjektive Anpassung von statistischen Prognosen bzw. die subjektive Einschätzung durch Experten besser sein kann. Unterschiede ergeben sich hauptsächlich durch die zu prognostizierende Zeitreihen und die Art der Informationen, nicht durch Unterschiede in den Individuen (Mathews und Diamantopoulos 1989). Des Weiteren führt eine nutzergerechte Aufbereitung der Informationen, wie bspw. Prognoseintervalle anstatt Punktschätzungen (Baginski et al. 1993; Pownall et al. 1993), zu besseren Entscheidungen (Johnson 1982). Jedoch reicht dies nicht aus, um Biases durch Heuristiken vollständig auszuschließen. Insbesondere der übersteigerte Optimismus (Overconfidence) ist in diesem Kontext zu nennen, da die Prognose von Intervallen die Entscheidungsperformance negativ beeinflussen kann (Bolger und Önkal-Atay 2004). Des Weiteren ist auch das Feedback-Learning eine wichtige Methode um Konsistenz, Anpassung und Genauigkeit der Schätzung zu befördern (Remus et al. 1996). Das FeedbackLearning trägt auch dazu bei, fehlerhafte Heuristiken und Biases auszugleichen, so dass die Prognoseperformance von Experten an die von statistischen Methoden heranreicht (Bolger und Önkal-Atay 2004; Goodwin et al. 2004). Jedoch kann nicht ausgeschlossen werden, dass auch andere Biases im Praxisumfeld trotz FeedbackLearning auftreten (Lawrence et al. 2006).

Dabei ist die Untersuchung psychologischer Phänomene nicht der allgemeinen Betriebswirtschaftslehre und der Psychologie vorbehalten. Die vielen Besonderheiten der Assetklasse Immobilien machen auch eine Erforschung des immobilienwirtschaftlichen Verhaltens erforderlich, das im nächsten Kapitel analysiert wird.

\section{Ausrichtung der verhaltensorientierten Immobilienforschung}

Unsere Recherche teilt sich in eine bibliographische, eine Entwicklungs- und eine methodische Analyse. Die bibliographische Analyse gibt einen Überblick darüber, wie sich die Stichprobe im Hinblick auf publizierende Journale, Größe der Forscherteams, sowie internationaler und praxisorientierter Beteiligung zusammensetzt. Die Entwicklungsanalyse resümiert die Ergebnisse und die Ausgewogenheit über die diversen immobilienwirtschaftlichen Disziplinen. Die methodische Untersuchung der Artikel soll Aufschluss über die eventuellen Forschungslücken geben. 


\subsection{Bibliographische Angaben}

Für die systematische Literaturanalyse wurden 48 international publizierte Artikel identifiziert. Insgesamt 39 Artikel wurden in immobilienwirtschaftlichen Zeitschriften publiziert, was einem Anteil von ca. 0,7\% aller in den Journalen publizierten Artikeln entspricht (siehe Tab. 1). Vergleicht man diese Zahlen mit den Ergebnissen von Arnott und Pervan (2005) erscheint diese Zahl gering, obwohl dies vorsichtig interpretiert werden sollte. Die Mehrheit dieser Publikationen wurde von zwei oder mehr Autoren erstellt, die am häufigsten aus zwei Institutionen kommen. Der Anteil an internationalen Forschungsteams beträgt ca. $17 \%$, und die Beteiligung nichtakademischer Einrichtungen liegt bei $10 \%$. Dabei ist auch interessant zu sehen, dass ca. $81 \%$ der Artikel aus der Immobilienwirtschaft und ca. 19\% der Fachbeiträge aus anderen Fachbereichen stammen. Dabei bildet sich kein einheitliches Medium heraus, sondern die Artikel sind verstreut über unterschiedliche Zeitschriften. Die Artikel von Diekmann et al. (1996) sowie Northcraft und Neale (1987) sind insofern interessant, da sie vermutlich als Katalysatoren für den Hauptteil der verhaltensorientierten Forschung innerhalb der Immobilienforschung dienten. Die restlichen Artikel scheinen zufällig oder durch eine breitere gesellschaftliche Thematik zustande gekommen zu sein (Bucchianeri und Minson 2013; Seiler et al. 2012, 2008; Genesove und Mayer 2001). Innerhalb der immobilienwirtschaftlichen Zeitschriften bildet sich das Journal of Property Investment and Finance als primäres Publikationsmedium für die verhaltensorientierte Forschung heraus, gefolgt vom Journal of Property Research. Die Diskussion findet somit nicht in den Journalen mit dem höchsten „Impact Factor“ wie Real Estate Economics, Real Estate Research oder Journal of Real Estate Finance and Economics statt (siehe Tab. 1 und auch Tab. 7 im Anhang A).

\subsection{Entwicklung des Forschungsstands}

Die Auszählung der Artikel erfolgt nach Verhaltensaspekten und immobilienwirtschaftlichen Themen. Wir haben die Themengebiete Projektentwicklung, Bewertung und Erwerb von Immobilien gewählt. Hinzu kommen eine allgemeine Kategorie und die Prognose von Immobilienmärkten als Sonderthema der Bereiche Bewertung und Investition, wobei Letztere auch Parallelen zum ,Judgmental Forecasting“ in der verhaltensorientierten Ökonomie aufweist (siehe Abschn. 3). Die beliebtesten Themen sind der Erwerb und die Bewertung von Immobilien, die einen Anteil von ca. $80 \%$ der gesamten identifizierten Literatur ausmachen (siehe Tab. 2). ${ }^{8}$ Ein Grund dafür ist vermutlich, dass Investitions- und Bewertungsentscheidungen ein numerisch fassbares, eindeutiges Ergebnis haben, was die Untersuchung des Verhaltens erleichtert. ${ }^{9}$ Jedoch sollte dies nicht dazu führen, dass eine Erforschung der weiteren in Tab. 2 aufgeführten Themengebiete vernachlässigt wird. Auch für den Untersuchungsgegenstand bilden sich klare Präferenzen unter den Forschungsteams heraus.

\footnotetext{
${ }^{8}$ Meyer und Pfnür (2015) untersuchen kognitive Verzerrungen für die Projektentwicklung in der Immobilienwirtschaft.

9 Siehe auch Lifka (2009) für einen breiten Überblick über Entscheidungsmethoden.
} 
Tab. 1 Bibliographische Auswertung verhaltensorientierter Immobilienforschung von 1989-2016

\begin{tabular}{|c|c|c|c|c|c|c|c|c|c|}
\hline \multirow{2}{*}{$\begin{array}{l}\text { Name des Jour- } \\
\text { nals }\end{array}$} & \multicolumn{3}{|c|}{ Artikel } & \multicolumn{2}{|c|}{ Forscherteam } & \multicolumn{2}{|c|}{ International } & \multicolumn{2}{|c|}{ Praxisbeteiligung } \\
\hline & Anz. & Insg. & $\%$ & Aut. & Inst. & Anz. & $\%$ & Anz. & $\%$ \\
\hline \multicolumn{10}{|c|}{ Immobilienwirtschaftliche Zeitschriften } \\
\hline $\begin{array}{l}\text { Int. Real Estate } \\
\text { Review }\end{array}$ & 1 & 177 & 0,6 & 4 & 2 & 1 & 100 & 0 & 0 \\
\hline $\begin{array}{l}\text { J. of Euro. Real } \\
\text { Estate Research }\end{array}$ & 3 & 126 & 2,4 & 3 & 2 & 1 & 33 & 1 & 33 \\
\hline $\begin{array}{l}\text { J. of Fin. Mgmt. } \\
\text { of Prop. \& Con }\end{array}$ & 1 & 181 & 0,6 & 2 & 1 & 0 & 0 & 0 & 0 \\
\hline J. of Prop. Fin & 1 & 204 & 0,5 & 3 & 1 & 0 & 0 & 0 & 0 \\
\hline $\begin{array}{l}\text { J. of Prop. Inv. \& } \\
\text { Fin }\end{array}$ & 15 & 548 & 2,7 & 2 & 2 & 2 & 13 & 2 & 13 \\
\hline $\begin{array}{l}\text { J. of Prop. Re- } \\
\text { search }\end{array}$ & 6 & 453 & 1,3 & 2 & 1 & 1 & 17 & 0 & 0 \\
\hline $\begin{array}{l}\text { J. of Prop. Val. \& } \\
\text { Inv }\end{array}$ & 3 & 187 & 1,6 & 2 & 1 & 0 & 0 & 0 & 0 \\
\hline $\begin{array}{l}\text { J. of Real Estate } \\
\text { Fin. \& Econ }\end{array}$ & 2 & 1179 & 0,2 & 3 & 2 & 0 & 0 & 1 & 50 \\
\hline $\begin{array}{l}\text { J. of Real Estate } \\
\text { Pract. \& Edu }\end{array}$ & 1 & 184 & 0,5 & 2 & 2 & 0 & 0 & 0 & 0 \\
\hline $\begin{array}{l}\text { J. of Real Estate } \\
\text { Research }\end{array}$ & 2 & 802 & 0,2 & 3 & 3 & 1 & 50 & 0 & 0 \\
\hline $\begin{array}{l}\text { Pacific Rim Prop. } \\
\text { Research J }\end{array}$ & 1 & 349 & 0,3 & 2 & 2 & 0 & 0 & 0 & 0 \\
\hline $\begin{array}{l}\text { Property Manage- } \\
\text { ment }\end{array}$ & 1 & 665 & 0,2 & 1 & 2 & 0 & 0 & 1 & 100 \\
\hline $\begin{array}{l}\text { Real Estate Eco- } \\
\text { nomics }\end{array}$ & 2 & 782 & 0,3 & 2 & 2 & 0 & 0 & 0 & 0 \\
\hline Zwischensumme & 39 & 5837 & 0,7 & 2 & 2 & 6 & 15 & 5 & 13 \\
\hline \multicolumn{10}{|l|}{ Andere Zeitschriften } \\
\hline Foresight & 1 & - & - & 1 & 1 & 0 & 0 & 0 & 0 \\
\hline $\begin{array}{l}\text { J. of Behav. \& } \\
\text { Exp. Econ }\end{array}$ & 1 & - & - & 2 & 2 & 0 & 0 & 0 & 0 \\
\hline J. of Behav. Fin & 2 & - & - & 3 & 2 & 0 & 0 & 0 & 0 \\
\hline $\begin{array}{l}\text { J. of Econ. Behav. } \\
\& \text { Org }\end{array}$ & 1 & - & - & 3 & 1 & 0 & 0 & 0 & 0 \\
\hline $\begin{array}{l}\text { Managerial \& } \\
\text { Dec. Econ }\end{array}$ & 1 & - & - & 1 & 1 & 0 & 0 & 0 & 0 \\
\hline $\begin{array}{l}\text { Org. Behav. \& } \\
\text { Hum. Dec. Proc }\end{array}$ & 2 & - & - & 4 & 3 & 1 & 50 & 0 & 0 \\
\hline $\begin{array}{l}\text { Quarterly J. of } \\
\text { Econ }\end{array}$ & 1 & - & - & 2 & 2 & 1 & 100 & 0 & 0 \\
\hline Gesamtsumme & 48 & - & - & 2 & 2 & 8 & 17 & 5 & 10 \\
\hline
\end{tabular}

Anz. Anzahl, Insg. Insgesamt, Aut. Autoren, Inst. Institutionen 
Tab. 2 Auswahl des Untersuchungsgegenstands in Abhängigkeit vom Themengebiet

\begin{tabular}{llllllll}
\hline $\begin{array}{l}\text { Themengebiet } \rightarrow \\
\begin{array}{l}\text { Untersuchungs- } \\
\text { gegenstand }\end{array}\end{array}$ & $\begin{array}{l}\text { All- } \\
\text { gemein }\end{array}$ & $\begin{array}{l}\text { Bewer- } \\
\text { tung }\end{array}$ & & $\begin{array}{l}\text { Erwerb von Immobilien } \\
\text { zur ... } \\
\text { Investition }\end{array}$ & $\begin{array}{l}\text { Prog- } \\
\text { Eigennutzung }\end{array}$ & $\begin{array}{l}\text { Projekt- } \\
\text { entwick- } \\
\text { lung }\end{array}$ & Gesamt \\
\hline $\begin{array}{l}\text { Begrenzt-rationale } \\
\text { Entsch }\end{array}$ & - & - & 1 & - & - & - & 1 \\
$\begin{array}{l}\text { Charaktergetriebene } \\
\text { Entsch }\end{array}$ & - & - & 1 & - & - & - & 1 \\
Entscheidungsmuster & - & 1 & - & - & - & - & 1 \\
Entscheidungskriterien & - & - & 3 & - & - & - & 3 \\
Entscheidungsprozess & - & 2 & 9 & - & 1 & 2 & 14 \\
Gruppenentscheidungen & - & - & - & - & 1 & - & 1 \\
\hline Zwischensumme & 0 & 3 & 14 & 0 & 2 & 2 & 21 \\
\hline Allgemein & 1 & 2 & - & - & - & - & 3 \\
Anchoring bias & - & 6 & 4 & 2 & - & - & 12 \\
Confirmation bias & - & 1 & - & - & - & - & 1 \\
Framing bias & - & - & 1 & - & - & - & 1 \\
Herding behavior & - & - & 1 & - & - & - & 1 \\
Loss aversion & - & - & 1 & 1 & - & - & 2 \\
Mental accounting & - & - & 1 & - & - & - & 1 \\
Moral hazard & - & 1 & - & - & - & - & 1 \\
Overconfidence & - & - & - & - & - & 1 & 1 \\
Regret aversion & - & - & 2 & - & - & - & 2 \\
Sunk cost effect & - & - & - & 1 & - & - & 1 \\
\hline Zwischensumme & 1 & 10 & 10 & 4 & 0 & 1 & 26 \\
\hline Ergebnis-Feedback & - & 1 & - & - & - & - & 1 \\
\hline Gesamtsumme & 1 & 14 & 24 & 4 & 2 & 3 & 48 \\
\hline & & & & & & & \\
\hline
\end{tabular}

Die meisten Untersuchungen liegen für den allgemeinen Entscheidungsprozess und die Ankerheuristik vor. Studien über das Lernen durch Erfahrung bzw. das FeedbackLearning werden in keinem Artikel behandelt. Lediglich Sah (2011) sowie Jackson und Orr (2011) präsentieren Ergebnisse über die Art der Informationsverarbeitung und deren Gewichtung durch Experten. Dies ist verwunderlich, da Cypher und Hansz (2003) deutliche Unterschiede bei der Informationsverarbeitung zwischen Laien und Experten feststellen. Lediglich Hansz und Diaz III. (2001) behandeln eine Form des Feedback-Prozesses. Jedoch ist diese Interpretation auf die soziale Interaktion anstatt auf das Lernen durch Erfahrung ausgerichtet. Die Überlegung folgt den Ergebnissen von Cho und Megbolugbe (1996), die die Überbewertung von Immobilien auf antrainiertes Sozialverhalten der Wertermittler zurückführen, um Diskussionen mit dem Auftraggeber über den Marktwert aus dem Weg zu gehen.

\subsection{Auswahl der Methodik}

Der Großteil der Forschungsartikel folgt dem üblichen ,positivistischen“ (naturwissenschaftlichen) Ansatz zur Untersuchung des Forschungsgegenstands. Im Ge- 
Tab. 3 Auswahl des Untersuchungsgegenstands in Abhängigkeit von der Forschungsmethode

\begin{tabular}{|c|c|c|c|c|c|c|c|c|}
\hline \multirow{2}{*}{$\begin{array}{l}\text { Forschungsmethode } \rightarrow \\
\downarrow \text { Untersuchungs- } \\
\text { gegenstand }\end{array}$} & \multicolumn{2}{|c|}{ Experiment } & \multicolumn{2}{|c|}{ Befragung } & \multicolumn{3}{|c|}{ Beobachtung } & \multirow[b]{2}{*}{ Gesamt } \\
\hline & Quasi & Voll & $\begin{array}{l}\text { Frage- } \\
\text { bogen }\end{array}$ & Interview & Fallstudie & $\begin{array}{l}\text { Markt- } \\
\text { daten }\end{array}$ & Sonst. & \\
\hline $\begin{array}{l}\text { Gebundene Rationa- } \\
\text { lität }\end{array}$ & - & - & 1 & - & - & - & - & 1 \\
\hline Gruppendynamik & - & 1 & - & - & - & - & - & 1 \\
\hline Gedankenmuster & 1 & - & - & - & - & - & - & 1 \\
\hline Entscheidungskriterien & 1 & 1 & 1 & - & - & - & - & 3 \\
\hline Entscheidungsprozess & - & 2 & 5 & 3 & 1 & - & 3 & 14 \\
\hline Persönlichkeitsmuster & 1 & - & - & - & - & - & - & 1 \\
\hline Zwischensumme & 3 & 4 & 7 & 3 & 1 & 0 & 3 & 21 \\
\hline Allgemein & - & - & - & - & - & - & 3 & 3 \\
\hline Anchoring bias & 1 & 9 & - & - & - & 2 & - & 12 \\
\hline Confirmation bias & - & - & 1 & - & - & - & - & 1 \\
\hline Framing bias & 1 & - & - & - & - & - & - & 1 \\
\hline Herding behavior & - & - & - & - & - & 1 & - & 1 \\
\hline Loss aversion & - & - & - & - & - & 2 & - & 2 \\
\hline Mental accounting & - & 1 & - & - & - & - & - & 1 \\
\hline Moral hazard & - & - & - & - & - & 1 & - & 1 \\
\hline Overconfidence & - & - & - & - & - & 1 & - & 1 \\
\hline Regret aversion & 1 & 1 & - & - & - & - & - & 2 \\
\hline Sunk cost effect & - & 1 & - & - & - & - & - & 1 \\
\hline Zwischensumme & 3 & 12 & 1 & 0 & 0 & 7 & 3 & 26 \\
\hline Ergebnis-Feedback & - & 1 & - & - & - & - & - & 1 \\
\hline Gesamtsumme & 6 & 17 & 8 & 3 & 1 & 7 & 6 & 48 \\
\hline
\end{tabular}

gensatz dazu existieren vier Artikel, die eine ,anti-positivistische“ Sichtweise einnehmen und detaillierte Einblicke in den Investmentprozess liefern (Reddy et al. 2014; Öhman et al. 2013; Gallimore und McAllister 2004; Gallimore et al. 2000). ${ }^{10}$ Die Einteilung in Positivismus und Anti-Positivismus soll die Auswirkung auf die Art der Ergebnisse betonen. Beide Gedankenschulen haben ihre Daseinsberechtigung und bringen Vor- und Nachteile mit sich. Daher bietet sich eine ausgewogene Kombination in einer größeren Forschungsagenda durchaus an. Allerdings ist das Verhältnis beider Ansätze in der analysierten Literatur sehr unausgewogen.

Die benötigte Vielfalt an Methoden wird deutlich, sobald eine Gesamtschau bestehender Ergebnisse vorgenommen wird (siehe Tab. 3). Parker (2014) verwendet semistrukturierte Interviews, um einen deskriptiven Entscheidungsprozess australischer REIT-Investoren abzuleiten. Roberts und Henneberry (2007) verwenden hingegen Fallstudien zweier konkurrierender Investoren. Beide Aufsätze geben interessante Einblicke in den Entscheidungsprozess von Investoren. Sollen hingegen Abweichun-

\footnotetext{
10 Es wird der Einteilung von Arnott und Pervan (2005) gefolgt, die die Forschungsmethoden im Bereich EUS in die Kategorien Positivismus, Anti-Positivismus und Methoden-Mix einteilen. Zweck ist eine Identifikation der grundsätzlichen Gedankenschule, die in erster Linie bewertungsfrei erfolgt, aber einen Einfluss auf die Art der Forschungsergebnisse hat.
} 
gen von einem rationalen Entscheidungsprozess untersucht werden, ist die erstere Methodik problematisch. French (2001) macht deutlich, dass das Gesagte (z.B. in einem Interview) von dem tatsächlich Umgesetzten abweicht. Auch Öhman et al. (2013) stellen klar,

[... that] investor surveys may give the impression that [...] issues in property investment can be reported in a check-list, whereas [...] such an approach is probably somewhat naive.

Tab. 3 führt die untersuchten Studien nach Untersuchungsgegenstand und Forschungsmethode auf. Die meisten Studien setzen Befragungen ein. Zwei Aufsätze befassen sich mit dem Entscheidungsprozess in der Bewertung (Diaz et al. 2004; Lin und Chang 2012) und verwenden Experimente. Alle Studien außer Parker (2014) kommen zu dem Ergebnis, dass der Entscheidungsprozess von einem rationalen Ideal abweicht bzw. der Prozess verkürzt wird.

Ein Teil der Studien führt diese Abweichungen auf Biases und Heuristiken zurück, die auch in Entscheidungsprozessen der Immobilienwirtschaft gefunden wurden, und zwar sowohl in Experimenten als auch mittels Marktdaten. In der Immobilienbewertung kommen Heuristiken ebenfalls vor, aber diese Problemstellung hält noch eine weitere Kuriosität bereit. Sie äußert sich u. a. darin, dass Laien-Gutachter den Wert häufiger und stärker auf- als abwerten (Havard 1999). Anders bei Experten-Gutachtern, denn hier scheinen der Kontext der Informationen sowie das Wissen und die Erfahrung ausschlaggebend zu sein. Die Anker fallen stärker aus, wenn der Gutachter den entsprechenden Immobilienmarkt nicht kennt (Cypher und Hansz 2003). Dafür übergewichten Gutachter die aktuellsten Informationen zu stark (Gallimore 1994) und suchen nach Informationen, um voreilige Einschätzungen zu bestätigen (Gallimore 1996). Des Weiteren scheint auch das „Appraisal Smoothing “ aufgrund kognitiver Verzerrungen zu entstehen (Gallimore und Wolverton 1997).

$\mathrm{Ob}$ solche Abweichungen durch Heuristiken entstehen, ist dabei nicht eindeutig zu beantworten. Öhman et al. (2013) weisen ähnlich wie Cho und Megbolugbe (1996) darauf hin,

that deviations from the normative literature and the prescribed rationality can

be seen as resulting from the [social] dynamics of the working group.

Daher kann auch die Frage gestellt werden, ob ein normativer Entscheidungsprozess überhaupt zielführender wäre und der Begriff ,Irrationalität“ bei Abweichungen sogar irreführend ist. Aus diesem Grund betonen Öhman et al. (2013) auch:

[... that] we need to enlarge our arsenal of analytical methods.

Eine ähnliche Problematik zeichnet sich auch bei der Gegenüberstellung von Experimenten und Marktdaten ab. Menschen verwenden bei Investitionen unterbewusst die Ankerheuristik (Black und Diaz III. 1996; Bucchianeri und Minson 2013), die zu fehlerhaften Einschätzungen führen kann. Im Gegensatz zur Bewertung scheint dieses Verhalten nicht unabhängig vom Erfahrungsgrad des Individuums aufzutreten (Black 1997; Genesove und Mayer 2001). Allerdings sind auch hier die Ergebnisse nicht als eindeutig einzustufen. Ling et al. (2016) präsentieren Ergebnisse, die die Suchkosten für Marktteilnehmer als Grund für Abweichungen anführen, der 
Ankerheuristik hingegen nur eine untergeordnete Rolle beimessen. Auch Bokhari und Geltner (2011) kommen zu diesem Schluss, weisen aber darauf hin, dass diese psychologischen Phänomene eine wesentliche Rolle auf Objektebene spielen. Zusammenfassend ergibt sich das Bild, dass bei Experimenten die Ankerheuristik stark und bei Marktbeobachtungen eher gering ausfällt. Während ökonometrische Modelle diejenigen Gleichungen bevorzugen, die am flexibelsten auf das Phänomen reagieren, provozieren Experimente das Phänomen in ,unnatürlich“ starker Weise. Daher warnt Diaz III. (1999) davor, die Ergebnisse zu stark zu generalisieren. Des Weiteren werden diese Phänomene immer in Abhängigkeit eines ,,instrumentellen“ Risikobegriffs betrachtet. Der Risikobegriff, so French und French (1997), ist aber unpräzise formuliert, denn Risiko umfasst wesentlich mehr als ein quantitatives Risikomaß auszudrücken vermag. Diese tiefergehende Problematik verdeutlicht, dass ein breiteres Spektrum an Methoden notwendig ist.

\section{Einführung in die Forschung zu Entscheidungsunterstützungs- systemen}

Ein erfolgreiches EUS muss nach den bisherigen Erkenntnissen drei unterschiedliche Aspekte berücksichtigen. Erstens muss die Effektivität des Systems gewährleistet werden, was ohne eine aktive Prüfung nicht möglich ist. Zweitens hängt die Effektivität maßgeblich von der Kognition des Nutzers und der Interaktion mit dem System ab, unabhängig davon ob psychologische Erkenntnisse in die Entwicklung einbezogen werden. Des Weiteren ist die Erfahrung des Nutzers wichtig, die sowohl in einem lernpsychologischen als auch in einem kognitionspsychologischen Kontext betrachtet werden sollte. Drittens muss sich das Arrangement der diversen Interventionsmaßnahmen mit der wahrgenommenen Problemstellung des Nutzers decken, denn die Akzeptanz ist eine Grundvoraussetzung für den Einsatz dieser Interventionen.

Die psychologischen Erkenntnisse deuten darauf hin, dass Biases einen maßgeblichen Einfluss auf die Effektivität eines EUS haben können und dies u.a. von der Informationsdarstellung abhängig ist. Eine Antwort der Wissenschaft auf diese Problemstellung ist die Entwicklung von Interventionen zur Verbesserung der Effektivität des EUS. Die Psychologie unterscheidet dabei zwei Kategorien zur Minderung kognitiver Verzerrungen: Änderung der Aufgabenstellung und Änderung des Nutzers. Das erste Feld versucht die Aufgabe so anzupassen, dass suboptimalen Entscheidungen vorgebeugt werden. Beispielsweise konnten Gigerenzer und Hoffrage (1995) in einem Experiment nachweisen, dass die Bayes-Inferenz durch Darstellung der relativen Häufigkeiten wesentlich einfacher für Probanden zu verstehen ist als durch Darstellung der Wahrscheinlichkeiten. Das zweite Feld hat in der Forschung eine wesentlich höhere Aufmerksamkeit bekommen. An dieser Stelle sollen zwei Strategien exemplarisch erwähnt werden. Die erste Strategie ist das Unpacking, das eine komplexe Entscheidung in einzelne Teile zerlegt und besser abschätzbar macht (Soll und Klayman 2004). Es ist primär auf das Phänomen Overconfidence ausgerichtet, das sich in systematischen Unterschätzungen von Probanden äußert. Ein Beispiel hierfür ist das übersteigerte Selbstbewusstsein von Menschen in die eigenen 
Tab. 4 Beispiele für nicht-computergestützte Strategien zur Minderung kognitiver Verzerrungen

\begin{tabular}{lll}
\hline Typ der Strategie & Name der Strategie & Quelle \\
\hline Änderung des & Consider-the-Opposite & Lord et al. (1984) \\
Nutzers & Prospective Hindsight & Mitchell et al. (1989) \\
& Dialectical Bootstrapping & Herzog und Hertwig (2009) \\
& Actor-Observer-Difference & Harvey et al. (1997) \\
& Cognitive Mapping & Hodgkinson et al. (1999) \\
& Unpacking & Soll und Klayman (2004) \\
Änderung der & Grafische Repräsentation & U.a. Lawrence et al. (2006) \\
Aufgabe & Informationsdarstellung & U.a. Gigerenzer und Hoffrage (1995) \\
\hline
\end{tabular}

Einschätzungen (Tversky und Kahneman 1974). Die zweite Strategie ist Considerthe-Opposite, bei der Individuen eine Aufgabe bekommen und nach Erledigung ihre Einschätzungen revidieren sollen (Lord et al. 1984). Diese Strategie soll insbesondere die Ankerheuristik mildern, die allerdings sehr persistent in Bezug auf ein solches Debiasing reagiert, wobei die Gründe hierfür noch unbekannt sind. Die Tab. 4 gibt eine Übersicht über diverse Strategien samt einem ausgewählten Quellenhinweis.

Das Thema Entscheidungsunterstützung wurde im Bereich der Informationstechnik unter Mitwirkung von Praktikern und Psychologen geboren. Daher unterscheidet sich die Auffassung zur Entscheidungsunterstützung von den zuvor vorgestellten Strategien. Power et al. (2011) definieren eine Entscheidungsunterstützung wie folgt:

[...] [A] DSS is a class of computerized information systems that support decision-making activities [...] which enhance users' effectiveness in making complex decisions $[\ldots]$.

Zum einen werden Informationssysteme in die Problemlösung einbezogen, was weitere Möglichkeiten für Interventionen ermöglicht, aber im gleichen Zuge auch die Komplexität erhöht. Zum anderen wird der Begriff „User Effectiveness “ eingeführt, der eng mit dem Begriff Entscheidungsqualität zusammenhängt. Anfangs wurde nur die Zufriedenheit des Nutzers anekdotenhaft oder in kurzen Fallbeschreibungen behandelt (Sharda et al. 1988). Ob das EUS wirklich wirksam den Nutzer bei seiner Entscheidungsfindung unterstützt, war ungeklärt und führte zur Entwicklung von entsprechenden Tests (siehe z. B. Ben-Zvi 2009). Es wurde auch bestätigt, dass EUS einen positiven Einfluss auf den Entscheidungsprozess von Probanden haben (Sainfort et al. 1990; Sharda et al. 1988). Jedoch gibt Udo (1992) zu bedenken, dass diese Aussage nicht generalisiert werden kann und stellt in seiner Literaturanalyse heraus, dass die Effektivität geprüft werden sollte. Ein möglicher Grund für Erfolg oder Misserfolg eines EUS scheint das Zusammenspiel von Aufgaben und bereitgestellten Informationen zu sein (Webby und O'Connor 1994; Montazemi et al. 1996), wie es bereits in der Psychologie zum Feedback-Learning festgestellt wurde.

Ein weiter Punkt ist, dass ein EUS nur effektiv den Nutzer unterstützen kann, wenn der Nutzer das System in seinem Lösungsprozess auch akzeptiert (Barr und Sharda 1997). Die Diskussion über die Akzeptanz von Informationssystemen wird nicht speziell für EUS geführt. Aber dieser Faktor ist vielleicht sogar wichtiger als bei anderen Informationssystemen, da es die Voraussetzung für die Effektivität 
des Systems darstellt. Daher sollte auch nicht von einer Ersetzung der Nutzerzufriedenheit durch die Effektivitätsmessung gesprochen werden, sondern über eine notwendige Ergänzung des Konzepts. Ein grundsätzliches Problem bei der Überprüfung der Nutzerakzeptanz ist auch hier die Erhebung. Ajzen (1991) stellt fest, dass die Korrelation zwischen Einstellung, beabsichtigtem Verhalten und tatsächlich beobachtetem Verhalten unzuverlässig sein kann. Daher entwickelt Ajzen die Theorie des geplanten Verhaltens, die eine Einschätzung des tatsächlichen menschlichen Handels verbessert. Venkatesh und Bala (2008) übertragen dieses Konzept in ihr Technology Acceptance Model (TAM). Des Weiteren existiert noch eine Integration in die allgemeine Nutzerzufriedenheit (Wixom und Todd 2005). Auch wenn das TAM nicht ohne Kritik ist (Bagozzi 2007), so liefert es dennoch umfangreiche Anhaltspunkte zur Optimierung von Systemen. Des Weiteren merken Greenhalgh et al. (2014) an, dass die Nutzung eines EUS auch von der Übereinstimmung des mentalen Modells und des EUS-Modells zur Problemlösung abhängig ist. Wenn die Art der Unterstützung inkompatibel mit der Herangehensweise des Nutzers ist, wird das EUS sehr wahrscheinlich nicht genutzt werden. Dieses Detail verdeutlicht die bedeutende Verbindung zu den Kognitionswissenschaften.

Das Thema kognitiv-nutzerzentrierter EUS ist in unserer Stichprobe nur gering ausgeprägt, obwohl Konzepte verfügbar sind (siehe z. B. Kasper 1996) und die Effektivität damit gesteigert werden kann. Einige interessante Arbeiten zu diesem Thema existieren trotzdem. Todd und Benbasat (1992) fanden heraus, dass ein EUS in Verbindung mit der Nutzerinteraktion betrachtet werden muss, da für den Nutzer die Minimierung des Aufwands ebenso wichtig ist, wie die Erhöhung der Effektivität von Entscheidungen. Es scheint aber grundsätzlich möglich Heuristiken zu trainieren oder neue Heuristiken zu erlernen (bspw. Gigerenzer und Kurzenhäuser 2005). George et al. (2000) sowie Lausberg und Dust (2017) haben Warnhinweise in ein DSS eingearbeitet, um die Ankerheuristik zu mildern, wenn auch mit wenig Erfolg. Bhandari et al. (2008) präsentieren Ergebnisse, bei der kognitive Verzerrungen mittels Visualisierungen und Feedback gemindert werden können. Allerdings kommen diese Eingriffe nicht ohne Risiken, wie die nachfolgenden Artikel zeigen. Arnold et al. (2004) berichten über intelligente Agenten eines EUS, die kognitive Verzerrungen von Experten mindern und bei Laien verstärken. Chen und Koufaris (2015) weisen darauf hin, dass die Kognition des Nutzers ein entscheidendes Kriterium beim Design eines EUS spielen sollte. Sie zeigen in ihrem Experiment, dass EUS die Risikobereitschaft von Laien stark erhöhen und zu verzerrten Entscheidungen führen. Kayande et al. (2009) berichten von besseren Entscheidungen, wenn das System gleichzeitig Feedback über das „Upside-Potential“"11 von und Verbesserungsvorschläge ${ }^{12}$ zu Entscheidungen bereitstellt. Diese Art des Feedbacks führt gleichzeitig auch zu einer hohen Zufriedenheit bei den Nutzern. Leider ist es die einzige Studie, die das MCPL-Paradigma in die EUS-Entwicklung einbezieht.

\footnotetext{
${ }^{11}$ Kayande et al. (2009) beschreiben Feedback zum „Upside-Potential“ wie folgt: Ein Verkäufer bekommt Informationen über seine Verkäufe relativ zu einer Benchmark, beispielsweise einer Gruppe mit den höchsten Verkaufszahlen.

12 Verbesserungsvorschläge können Informationen darüber sein, wie eine höhere Erfolgsrate beim Verkauf erzielt werden kann.
} 


\section{Ausrichtung der EUS-Forschung in der Immobilienwirtschaft}

Im Bereich der immobilienwirtschaftlichen EUS wurden 40 international publizierte Artikel identifiziert, wobei 13 Artikel aus immobilienwirtschaftlichen und 27 Artikel aus nicht-immobilienwirtschaftlichen Zeitschriften stammen (siehe Tab. 5 und auch Tab. 8 im Anhang B). Bezogen auf die immobilienwirtschaftlichen Journalartikel entspricht dies einem Anteil von 0,4\% aller in den erfassten immobilienwirtschaftlichen Zeitschriften veröffentlichten Beiträge. Innerhalb der immobilienwirtschaftlichen Journale kristallisiert sich im Gegensatz zur verhaltensorientierten Literatur (s. Tab. 1) auch kein favorisiertes Medium heraus. Die Artikel sind auf eine Vielzahl unterschiedlicher Journale verteilt, was einen wissenschaftlichen Diskurs erschweren kann. Weiterhin ist die Größe der Forschungsteams mit 2-3 Personen tendenziell größer, aber die Forscher kommen meistens aus der gleichen Institution. Die Internationalität der Teams ist gleich hoch wie bei der verhaltensorientierten Forschung, dennoch ist die Internationalität als eher gering zu betrachten. Die Beteiligung der Praxis fällt mit $25 \%$ wesentlich höher aus als im Bereich der verhaltensorientierten Immobilienforschung.

Für die Auswertung wurden die Verteilung über die jeweiligen Disziplinen sowie die drei zuvor beschriebenen Beurteilungskriterien Kognition, Effektivität und Nutzerakzeptanz erhoben (siehe Tab.6). Die Verteilung über die verschiedenen immobilienwirtschaftlichen Disziplinen deckt sich mit den Ergebnissen zur verhaltensorientierten Immobilienforschung. Danach ist die Mehrzahl der Artikel auf die Bereiche „Investment und Bewertung“ ausgerichtet. Die Popularität des Themas Bewertung ist vermutlich der Gesetzgebung geschuldet, die eine Massenbewertung von Immobilien und im Umkehrschluss solche Systeme notwendig macht. Ähnlich verhält es sich mit dem Thema „Investment“, was vielleicht mit der hohen Bedeutung der Herstellungs- und Akquisitionsphase zusammenhängt, die den Investmentver-

Tab. 5 Bibliographische Auswertung immobilienwirtschaftlicher EUS-Forschung von 1989-2016

\begin{tabular}{|c|c|c|c|c|c|c|c|c|c|}
\hline \multirow[t]{2}{*}{ Name des Journals } & \multicolumn{3}{|c|}{ Artikel } & \multicolumn{2}{|c|}{ Forscherteam } & \multicolumn{2}{|c|}{ International } & \multicolumn{2}{|c|}{ Praxisbet } \\
\hline & Anz. & Insg. & $\%$ & Anz. & Inst. & Anz. & $\%$ & Anz. & $\%$ \\
\hline \multicolumn{10}{|c|}{ Immobilienwirtschaftliche Zeitschriften } \\
\hline Con. Inno & 1 & 345 & 0,3 & 3 & 2 & 1 & 100 & 0 & 0 \\
\hline Int. J. of Str. Prop. Mgmt & 3 & 307 & 1,0 & 4 & 1 & 0 & 0 & 0 & 0 \\
\hline J. of Corp. Real Estate & 1 & 359 & 0,3 & 3 & 1 & 0 & 0 & 0 & 0 \\
\hline $\begin{array}{l}\text { J. of Fin. Mgmt. of Prop } \\
\text { \& Con }\end{array}$ & 1 & 181 & 0,6 & 4 & 3 & 0 & 0 & 1 & 100 \\
\hline J. of Housing Research & 2 & 158 & 1,3 & 2 & 2 & 0 & 0 & 2 & 100 \\
\hline J. of Prop. Inv. \& Fin & 2 & 548 & 0,4 & 2 & 1 & 0 & 0 & 1 & 50 \\
\hline $\begin{array}{l}\text { J. of Real Estate Re- } \\
\text { search }\end{array}$ & 1 & 802 & 0,1 & 3 & 2 & 0 & 0 & 0 & 0 \\
\hline Journal of Valuation & 1 & 39 & 2,6 & 1 & 1 & 0 & 0 & 0 & 0 \\
\hline $\begin{array}{l}\text { Pacific Rim Prop. Re- } \\
\text { search J }\end{array}$ & 1 & 349 & 0,3 & 2 & 2 & 0 & 0 & 0 & 0 \\
\hline $\begin{array}{l}\text { Zwischensumme bzw. } \\
\text { Mittelwert }\end{array}$ & 13 & 3088 & 0,4 & 3 & 2 & 1 & 8 & 4 & 31 \\
\hline
\end{tabular}


Tab. 5 (Fortsetzung)

\begin{tabular}{|c|c|c|c|c|c|c|c|c|c|}
\hline \multirow[t]{2}{*}{ Name des Journals } & \multicolumn{3}{|c|}{ Artikel } & \multicolumn{2}{|c|}{ Forscherteam } & \multicolumn{2}{|c|}{ International } & \multicolumn{2}{|c|}{ Praxisbet } \\
\hline & Anz. & Insg. & $\%$ & Anz. & Inst. & Anz. & $\%$ & Anz. & $\%$ \\
\hline \multicolumn{10}{|l|}{ Andere Zeitschriften } \\
\hline Astin Bulletin & 1 & - & - & 2 & 2 & 0 & 0 & 1 & 100 \\
\hline Decision Sciences & 1 & - & - & 1 & 1 & 0 & 0 & 0 & 0 \\
\hline $\begin{array}{l}\text { Decision Support Sys- } \\
\text { tems }\end{array}$ & 4 & - & - & 3 & 2 & 1 & 25 & 0 & 0 \\
\hline $\begin{array}{l}\text { Env. \& Plan. B: Planning } \\
\text { \& Design }\end{array}$ & 1 & - & - & 3 & 3 & 1 & 100 & 1 & 100 \\
\hline $\begin{array}{l}\text { Euro. J. of Operational } \\
\text { Research }\end{array}$ & 1 & - & - & 2 & 2 & 0 & 0 & 1 & 100 \\
\hline $\begin{array}{l}\text { Expert Systems with } \\
\text { Applications }\end{array}$ & 2 & - & - & 2 & 1 & 0 & 0 & 0 & 0 \\
\hline $\begin{array}{l}\text { Information \& Manage- } \\
\text { ment }\end{array}$ & 1 & - & - & 1 & 1 & 0 & 0 & 1 & 100 \\
\hline Intellectual Economics & 1 & - & - & 2 & 1 & 0 & 0 & 0 & 0 \\
\hline Interfaces & 1 & - & - & 1 & 1 & 0 & 0 & 1 & 100 \\
\hline Int. J. of Geo. Inf. Sc & 1 & - & - & 2 & 2 & 1 & 100 & 0 & 0 \\
\hline Int. J. of Geo. Inf. Sys & 1 & - & - & 1 & 1 & 0 & 0 & 0 & 0 \\
\hline $\begin{array}{l}\text { Int. J. of Inf. \& Com. } \\
\text { Tech. Research }\end{array}$ & 1 & - & - & 1 & 1 & 0 & 0 & 0 & 0 \\
\hline $\begin{array}{l}\text { Int. J. of Inf. Sec. \& Sys. } \\
\text { Mgmt }\end{array}$ & 1 & - & - & 3 & 1 & 0 & 0 & 0 & 0 \\
\hline $\begin{array}{l}\text { J. of Bus. \& Econ. Re- } \\
\text { search }\end{array}$ & 1 & - & - & 2 & 1 & 0 & 0 & 0 & 0 \\
\hline J. of Civ. Eng. \& Mgmt & 1 & - & - & 2 & 1 & 0 & 0 & 0 & 0 \\
\hline $\begin{array}{l}\text { J. of Emer. Trends in } \\
\text { Comp. \& Inf. Sc }\end{array}$ & 1 & - & - & 4 & 3 & 0 & 0 & 0 & 0 \\
\hline $\begin{array}{l}\text { J. of Theo. \& Appl. Inf. } \\
\text { Tech }\end{array}$ & 1 & - & - & 1 & 1 & 0 & 0 & 0 & 0 \\
\hline Omega & 1 & - & - & 1 & 1 & 0 & 0 & 0 & 0 \\
\hline $\begin{array}{l}\text { Proc. of the Inst. of } \\
\text { Mech. Eng }\end{array}$ & 1 & - & - & 2 & 2 & 0 & 0 & 0 & 0 \\
\hline Review of Econ. \& Fin & 1 & - & - & 1 & 1 & 0 & 0 & 0 & 0 \\
\hline Sc. J. of Riga Tech. Uni & 1 & - & - & 2 & 1 & 0 & 0 & 0 & 0 \\
\hline Socio-Econ. Planning Sc & 1 & - & - & 2 & 2 & 1 & 100 & 1 & 100 \\
\hline Trans. in Bus. \& Econ & 1 & - & - & 3 & 1 & 0 & 0 & 0 & 0 \\
\hline $\begin{array}{l}\text { Zwischensumme bzw. } \\
\text { Mittelwert }\end{array}$ & 27 & - & - & 2 & 1 & 4 & 15 & 6 & 22 \\
\hline $\begin{array}{l}\text { Gesamtsumme bzw. } \\
\text { Mittelwert }\end{array}$ & 40 & - & - & 2 & 1 & 5 & 13 & 10 & 25 \\
\hline
\end{tabular}


Tab. 6 Verteilung der Forschungsartikel auf Fachgebiete sowie Berücksichtigung der Beurteilungskriterien $^{\mathrm{a}}$

\begin{tabular}{llllll}
\hline Aufgabe & Artikel & & & & \\
& Anz & $\%$ & Effektivität & Kognition & Akzeptanz \\
\hline Bewertung & 11 & 28 & 0 & 1 & 0 \\
Corporate Real Estate & 1 & 3 & 0 & 0 & 0 \\
Facility Management & 1 & 3 & 0 & 0 & 0 \\
Finanzierung & 1 & 3 & 0 & 0 & 0 \\
Investmentmanagement & 9 & 23 & 0 & 0 & 0 \\
Öffentliche Verwaltung & 2 & 5 & 0 & 0 & 0 \\
Portfoliomanagement & 4 & 10 & 0 & 0 & 0 \\
Prognose & 3 & 8 & 1 & 0 & 0 \\
Projektentwicklung & 4 & 10 & 0 & 0 & 0 \\
Property Management & 2 & 5 & 0 & 0 & 0 \\
Risikomanagement & 2 & 5 & 0 & 0 & 0 \\
Gesamtsumme & 40 & 100 & 1 & 1 & 0 \\
\hline
\end{tabular}

${ }^{\mathrm{a}}$ Grundsätzlich könnte bei der Verteilung der Artikel auf die Fachbereiche von einer Gleichverteilung ausgegangen werden. Dies wäre der statistisch beste Schätzwert in Abwesenheit weiterer geeigneter Informationen. Da die Digitalisierung der Immobilienwirtschaft alle Bereiche betrifft, denn alle Bereiche nutzen in irgendeiner Weise IT-Systeme, ist das Ergebnis insofern interessant, da eine Häufung im Bereich „Bewertung und Investment" festgestellt werden kann

lauf maßgeblich beeinflusst. Zum anderen werden die Einflussfaktoren Effektivität, Kognition und Akzeptanz (fast) vollständig vernachlässigt. Dies deckt sich auch mit den Befunden der allgemeinen EUS-Forschung, die u.a. unter einer Ausrichtung mit geringer praktischer Relevanz leidet, sich über die Jahre aber verbessert hat (Arnott und Pervan 2014, 2008, 2005). Jedoch ist es nicht so, dass das Thema ganz vernachlässigt wird. Tidwell und Gallimore (2012) untersuchen die Einflüsse von kognitiven EUS auf die Bewertung von Immobilien. Sie kommen zu dem Schluss, dass ein EUS mit Debiasing den Ankereffekt reduzieren kann. Allerdings kann nicht abschließend geklärt werden, ob das Debiasing tatsächlich erfolgreich ist, da dies auch an der Informationsaufbereitung des EUS selbst liegen kann.

Evans et al. (2019) kommen in einer in Südafrika durchgeführten Untersuchung zu dem Schluss, dass Debiasing sich positiv auf die Bewertung auswirken kann. Allerdings können die Autoren eine Varianzverringerung bei der Marktwertermittlung statistisch nicht nachweisen. Sie weisen ferner darauf hin, dass die Unterschiede zwischen den Probanden eine große Bedeutung einnehmen und bei Untersuchungen berücksichtigt werden sollten. Dies passt zu den Aussagen von Diaz III. (1997) sowie Cypher und Hansz (2003) zur unterschiedlichen Stärke der Ankerheuristik bei Probanden. Lausberg und Dust (2017) kommen zu dem Schluss, dass das von Ihnen entwickelte EUS die Genauigkeit der Bewertung verbessert hat. Diese unterschiedlichen Ergebnisse könnten ein Hinweis auf kulturspezifische Heuristiken sein, wie sie auch von Diaz III. et al. (2004) berichtet werden. Trotz oder gerade wegen dieser interessanten Ergebnisse sollte die Forschung hier intensiviert werden, auch übergreifend zu anderen Disziplinen. Introne und Iandoli (2014) sind die einzigen Autoren, die auch die Effektivität des entwickelten Prognose-EUS nachweisen. Ein 
wichtiger Kernbestandteil, der bei Debiasing-Strategien automatisch als Ergebnis produziert wird, jedoch beim Gros der restlichen EUS-Artikel fehlt. Des Weiteren konnte keine Literatur zu lernpsychologischen Effekten wie dem Feedback-Learning gefunden werden, obwohl dies Auswirkungen auf die Stärke von Heuristiken haben kann (Bolger und Önkal-Atay 2004).

\section{Zusammenfassung, Bewertung und Ausblick}

Die vorausgegangene Literaturanalyse hatte zum Ziel, den aktuellen Forschungsstand $\mathrm{zu}$ eruieren und eventuelle Forschungslücken im Bereich immobilienwirtschaftlicher Entscheidungen zu identifizieren. Hierfür wurde eine vereinfachte systematische Literaturanalyse verwendet, die Ausprägungen der Forschungsartikel zu Merkmalen wie Aufgabenfeld, Methodik, Forschungsfokus, involvierte Journale, Autoren oder auch Beteiligung der Praxis erhebt. Die Auswertung hat dabei gleich mehrere Forschungslücken, unausgewogene Forschungsfelder sowie mangelnde Transferleistungen zwischen den Forschungsbereichen ergeben.

\subsection{Forschungslücken}

Insgesamt traten drei Kernbereiche in der Analyse hervor, die für die zukünftige Forschung einer intensiveren Diskussion bedürfen.

1. Die verhaltensorientierte Immobilienforschung hat in den letzten 30 Jahren Fortschritte im Bereich kognitiver Heuristiken und Verzerrungen gemacht. Leider wurde der Bereich der Lernpsychologie, im Speziellen das Feedback-Learning, nicht in die Forschung integriert. Des Weiteren existieren große Unterschiede zwischen dem Verhalten von Laien und Experten, die sehr unterschiedliche Ergebnisse hervorbringen können (siehe z. B. Arnold et al. 2004). Dieser Punkt wird in Untersuchungsdesigns bisher noch zu wenig beachtet; in vielen Experimenten werden weiterhin studentische Laien als Probanden herangezogen, was die Interpretation stark einschränkt.

2. Die Forschung beschäftigt sich fast ausschließlich mit der menschlichen Entscheidung unter Unsicherheit. Die meisten Experimente, aber auch EUS, integrieren quantitative Risikodefinitionen in den Versuchsanordnungen. Dies ist nicht überraschend, denn im Bereich der Risikoquantifizierung und Methodenentwicklung wurden die größten Fortschritte erzielt. Dennoch ist bekannt, dass die Auffassung und Wahrnehmung von Risiken stark vom instrumentellen Risikobegriff abweichen. Es ist notwendig, dass diese Begriffe in einem wissenschaftlichen Diskurs angenähert werden, und zwar in einer Weise, die es erlaubt, lern- und kognitionspsychologische Aspekte zu integrieren.

3. Es sollte sichergestellt werden, dass ein EUS effektiv den Nutzer unterstützt und sich nicht nachteilig auf die Entscheidungen auswirkt. Dies wird in dem überwiegenden Teil der Literatur außer Acht gelassen, was ein Gefahrenpotential für den Einsatz solcher Applikationen birgt. 


\subsection{Forschungstransfer und Balance}

Ein Grund für die Forschungslücken ist der fehlende Transfer von der allgemeinen verhaltensorientierten Forschung in die spezielle Betriebswirtschaftslehre der Immobilie. Des Weiteren ist auch der Transfer zwischen der verhaltensorientierten Immobilienforschung und der Forschung zu Immobilien-EUS kaum ausgeprägt. Ein Grund für diese Situation scheint zu sein, dass die Forscherteams aus dem EUS-Feld zu einem geringen Teil der Immobilienwirtschaft entstammen. Zusätzlich werden Publikationsmedien gewählt, die von der Immobilienforschung weniger frequentiert werden. Es wäre wünschenswert, dass sich internationale ImmobilienJournale als Diskussionsplattform etablieren, um den Transfer zwischen den Disziplinen zu fördern. Auch die Forschungsfelder sind wenig ausgewogen. Die Forschung konzentriert sich derzeit primär auf die Felder „Bewertung“ und „Investment“, vernachlässigt dabei aber alle anderen Bereiche wie Asset, Property und Facility Management, in denen ebenfalls wichtige Entscheidungen während des Bewirtschaftungszeitraums getroffen werden. Auch auf diesen Feldern besteht Potential für zukünftige Forschung.

\subsection{Limitationen}

Die Literaturanalyse konzentriert sich auf die international veröffentliche Literatur in wissenschaftlichen begutachteten Journalen. Aber nicht alle immobilienwirtschaftlichen Journale sind in den jeweiligen Datenbanken auffindbar bzw. lizensiert, so dass auch die Nutzung der Suchdatenbanken einen Filtereffekt in die Recherche integriert. Darüber hinaus ist das Suchergebnis von der Verschlagwortung der Artikel und dem Suchalgorithmus abhängig, so dass es sein kann, dass Artikel innerhalb der identifizierten Journale übersehen werden. Daher sollte die Recherche als eine Stichprobe angesehen werden, die in diesem Zusammenhang auch eine stichprobenbedingte Streuung aufweist. Bei der Interpretation der Ergebnisse sollte weiterhin berücksichtig werden, dass nicht alle wertvollen Forschungsergebnisse auch in wissenschaftlichen Zeitschriften veröffentlicht werden. Daher sind die hier präsentierten Ergebnisse mit Vorsicht zu verwenden.

\subsection{Ausblick}

Das Forschungsfeld der immobilienwirtschaftlichen EUS steht sowohl am Ende als auch ganz am Anfang seiner Entwicklung. Im klassischen Sinne ist die EUS-Forschung mit der methodischen Zusammenstellung von Analysemethoden ausgereizt und bietet kaum noch Potential für interessante Erkenntnisse. Arnott und Pervan (2014) sehen das als übergreifendes Problem der EUS-Forschung, auch wenn es Anzeichen für eine Besserung gibt. Allerdings sehen wir die EUS-Forschung in einem verhaltensorientierten Kontext aus drei Gründen noch ganz am Anfang.

1. Es sind noch viele Fragen über die menschliche Kognition und deren Interaktion mit Informationssystemen offen. 
2. Die Determinanten für ein effektives EUS sind ebenfalls nicht bekannt, aber für die Entwicklung solcher Systeme zwangsläufig notwendig. Die Forschung zeigt, dass die Effektivität eines EUS nicht angenommen werden kann.

3. Der Risikobegriff bedarf einer weiteren Diskussion. In diesem Kontext sind auch immobilienwirtschaftliche Kennzahlen zu überdenken. Insbesondere zukunftsgerichtete Risikokennzahlen sollten ein Anliegen der weiteren wissenschaftlichen Diskussion sein.

Shefrin (2016, S. 69) ist der Meinung:

There is little evidence to suggest that debiasing on one's own is likely to prove fruitful. Making inroads along the debiasing dimension requires group effort and can only take place in the right kind of social environment.

Auch wenn die Ergebnisse aus den bereits oben genannten Gründen (s. Limitationen) vorsichtig interpretiert werden müssen, scheint auf Basis unserer Stichprobe das Forschungsfeld kognitiv-nutzerzentrierter EUS Potential für Weiterentwicklungen und neue Themen zu bieten, so dass man auf die zukünftige Forschung gespannt sein darf.

Interessenkonflikt P. Krieger und C. Lausberg geben an, dass kein Interessenkonflikt besteht.

Open Access Dieser Artikel wird unter der Creative Commons Namensnennung 4.0 International Lizenz veröffentlicht, welche die Nutzung, Vervielfältigung, Bearbeitung, Verbreitung und Wiedergabe in jeglichem Medium und Format erlaubt, sofern Sie den/die ursprünglichen Autor(en) und die Quelle ordnungsgemäß nennen, einen Link zur Creative Commons Lizenz beifügen und angeben, ob Änderungen vorgenommen wurden.

Die in diesem Artikel enthaltenen Bilder und sonstiges Drittmaterial unterliegen ebenfalls der genannten Creative Commons Lizenz, sofern sich aus der Abbildungslegende nichts anderes ergibt. Sofern das betreffende Material nicht unter der genannten Creative Commons Lizenz steht und die betreffende Handlung nicht nach gesetzlichen Vorschriften erlaubt ist, ist für die oben aufgeführten Weiterverwendungen des Materials die Einwilligung des jeweiligen Rechteinhabers einzuholen.

Weitere Details zur Lizenz entnehmen Sie bitte der Lizenzinformation auf http://creativecommons.org/ licenses/by/4.0/deed.de. 


\section{Anhang A}

Tab. 7 Auflistung aller Artikel zur verhaltensorientierten Immobilienforschung

\begin{tabular}{|c|c|c|}
\hline Disziplin & Heuristik & Autor, Herausgeber oder Institution \\
\hline \multirow[t]{7}{*}{ Bewertung } & Anchoring bias & $\begin{array}{l}\text { Cypher und Hansz (2003), Diaz III. (1997), Gallimore (1994), Gal- } \\
\text { limore und Wolverton (1997), Havard (1999), Northcraft und Neale } \\
\text { (1987) }\end{array}$ \\
\hline & $\begin{array}{l}\text { Confirmation } \\
\text { bias }\end{array}$ & Gallimore (1996) \\
\hline & $\begin{array}{l}\text { Entscheidungs- } \\
\text { prozess }\end{array}$ & Diaz III. et al. (2004), Lin und Chang (2012) \\
\hline & Moral hazard & Cho und Megbolugbe (1996) \\
\hline & $\begin{array}{l}\text { Feedback- } \\
\text { Effekt }\end{array}$ & Hansz und Diaz III. (2001) \\
\hline & $\begin{array}{l}\text { Kognitions- } \\
\text { muster }\end{array}$ & Bellman und Öhman (2016) \\
\hline & Sonstiges & Diaz III. und Hansz (2007), Wofford et al. (2011), Diaz III. (1999) \\
\hline \multirow[t]{10}{*}{ Investment } & Anchoring bias & $\begin{array}{l}\text { Black und Diaz III. (1996), Diaz III. et al. (1999), Ling et al. (2016), } \\
\text { Lowies et al. (2016) }\end{array}$ \\
\hline & $\begin{array}{l}\text { Begrenzte } \\
\text { Rationalität }\end{array}$ & De Bruin und Flint-Hartle (2003) \\
\hline & $\begin{array}{l}\text { Entscheidungs- } \\
\text { kriterien }\end{array}$ & Adair et al. (1994), Jackson und Orr (2011), Sah (2011) \\
\hline & $\begin{array}{l}\text { Entscheidungs- } \\
\text { prozess }\end{array}$ & $\begin{array}{l}\text { Farragher und Savage (2008), French (2001), French und French } \\
\text { (1997), Gallimore et al. (2000), MacCowan und Orr (2008), Öhman } \\
\text { et al. (2013), Parker (2014), Reddy et al. (2014), Roberts und Henne- } \\
\text { berry (2007) }\end{array}$ \\
\hline & Framing bias & Ye und Dent (2009) \\
\hline & $\begin{array}{l}\text { Herding beha- } \\
\text { viour }\end{array}$ & Byrne et al. (2013) \\
\hline & $\begin{array}{l}\text { Mental ac- } \\
\text { counting }\end{array}$ & Seiler et al. (2012) \\
\hline & $\begin{array}{l}\text { Persönlichkeits- } \\
\text { merkmale }\end{array}$ & Ben-Shahar und Golan (2014) \\
\hline & $\begin{array}{l}\text { Regret aversi- } \\
\text { on }\end{array}$ & Seiler und Seiler (2010), Seiler et al. (2008) \\
\hline & Loss aversion & Bokhari und Geltner (2011) \\
\hline \multirow[t]{2}{*}{ Prognose } & $\begin{array}{l}\text { Entscheidungs- } \\
\text { prozess }\end{array}$ & Gallimore und McAllister (2004) \\
\hline & Gruppendynamik & Ratcliffe (2001) \\
\hline \multirow[t]{2}{*}{$\begin{array}{l}\text { Projekt- } \\
\text { entwicklung }\end{array}$} & $\begin{array}{l}\text { Entscheidungs- } \\
\text { prozess }\end{array}$ & Ahiaga-Dagbui und Smith (2014), Atherton et al. (2008) \\
\hline & Overconfidence & Wang et al. (2000) \\
\hline \multirow[t]{3}{*}{ Eigenheim } & Anchoring bias & Black (1997), Bucchianeri und Minson (2013) \\
\hline & $\begin{array}{l}\text { Sunk cost } \\
\text { effect }\end{array}$ & Diekmann et al. (1996) \\
\hline & Loss aversion & Genesove und Mayer (2001) \\
\hline
\end{tabular}




\section{Anhang B}

Tab. 8 Auflistung aller Artikel zu Immobilien-EUS

\begin{tabular}{|c|c|c|}
\hline $\begin{array}{l}\text { EUS } \\
\text { Typ }\end{array}$ & Disziplin & Autor, Herausgeber oder Institution \\
\hline \multirow[t]{2}{*}{ GDSS } & Investment & Cebi und Kahraman (2010), Urbanavičiene et al. (2009a), (2009b) \\
\hline & $\begin{array}{l}\text { Öffentliche } \\
\text { Verwaltung }\end{array}$ & Phillips et al. (2007) \\
\hline \multirow[t]{2}{*}{ KDSS } & $\begin{array}{l}\text { Facility Ma- } \\
\text { nagement }\end{array}$ & Han et al. (1991) \\
\hline & Investment & Zavadskas und Kaklauskas (2009) \\
\hline \multirow[t]{9}{*}{ PDSS } & Bewertung & $\begin{array}{l}\text { Greer und Murtaza (2003), Kaklauskas et al. (2007), Kettani und Khelifi } \\
\text { (2001), Kettani und Oral (2015), Lausberg und Dust (2017), Tidwell und } \\
\text { Gallimore (2012), Evans et al. (2019) }\end{array}$ \\
\hline & CRE & Hoffman et al. (1990) \\
\hline & Investment & Kaklauskas und Gikys (2005), Kaklauskas et al. (2001) \\
\hline & $\begin{array}{l}\text { Öffentliche } \\
\text { Verwaltung }\end{array}$ & Wang (2005) \\
\hline & $\begin{array}{l}\text { Portfolio } \\
\text { Management }\end{array}$ & Simoni (2011), Trippi (1989), Valverde (2010), Metzner (2013) \\
\hline & Prognose & Forgionne (1996), Introne und Iandoli (2014) \\
\hline & $\begin{array}{l}\text { Projekt- } \\
\text { entwicklung }\end{array}$ & $\begin{array}{l}\text { Leelarasamee (2005), Natividade-Jesus et al. (2007), Pommer (2007), } \\
\text { Han und Kim (1990) }\end{array}$ \\
\hline & $\begin{array}{l}\text { Property } \\
\text { Management }\end{array}$ & Van Reedt Dortland et al. (2012), Taillandier et al. (2014) \\
\hline & $\begin{array}{l}\text { Risiko- } \\
\text { management }\end{array}$ & Lowe und Stanard (1997), Valverde (2011) \\
\hline \multirow[t]{5}{*}{ SDSS } & Bewertung & McCluskey und Anand (1999) \\
\hline & Finanzierung & Belsky et al. (1998) \\
\hline & Investment & Peterson (1993), (1998), Podor und Nyiri (2010), Zeng und Zhou (2001) \\
\hline & $\begin{array}{l}\text { Projekt- } \\
\text { entwicklung }\end{array}$ & Coutinho-Rodrigues et al. (2011), Li et al. (2005), Arentze et al. (1996) \\
\hline & $\begin{array}{l}\text { Property } \\
\text { Management }\end{array}$ & Kisilevich et al. (2013) \\
\hline GES & Investment & HosseinKefi et al. (2013) \\
\hline \multirow[t]{3}{*}{ PES } & Bewertung & $\begin{array}{l}\text { Czernkowski (1990), Gonzalez und Laureano-Ortiz (1992), Kilpatrick } \\
\text { (2011), Larranz (2011), Moore (1992), Musa et al. (2013) }\end{array}$ \\
\hline & $\begin{array}{l}\text { Portfolio } \\
\text { Management }\end{array}$ & Ellis und Wilson (2006) \\
\hline & Prognose & Rossini (2000) \\
\hline Allgemein & - & Kaklauskas et al. (2009), Trippi (1990) \\
\hline
\end{tabular}




\section{Literatur}

Adair A, Hutchison N (2005) The reporting of risk in real estate appraisal property risk scoring. J Prop Invest Finance 23(3):254-268

Adair AS, Berry JN, McGreal WS (1994) Investment decision making: a behavioural perspective. J Prop Finance 5(4):32-42

Ahiaga-Dagbui DD, Smith SD (2014) Rethinking construction cost overruns: cognition, learning and estimation. J Financ Manag Prop Constr 19(1):38-54

Ajzen I (1991) The theory of planned behavior. Organ Behav Hum Decis Process 50(2):179-211

Arentze TA, Borgers AWJ, Timmermans HJP (1996) An efficient search strategy for site-selection decisions in an expert system. Geogr Anal 28(2):126-146

Arnold V, Collier PA, Leech SA, Sutton SG (2004) Impact of intelligent decision aids on expert and novice decision-makers' judgments. Account Financ 44(1):1-26

Arnott D (2006) Cognitive biases and decision support systems development: a design science approach. Inf Syst J 16(1):55-78

Arnott D, Pervan G (2005) A critical analysis of decision support systems research. J Inf Technol 20(2):67-87

Arnott D, Pervan G (2008) Eight key issues for the decision support systems discipline. Decis Support Syst 44(3):657-672

Arnott D, Pervan G (2014) A critical analysis of decision support systems research revisited: the rise of design science. J Inf Technol 29(4):269-293

Artzner P, Delbaen F, Eber J-M, Heath D (1999) Coherent measures of risk. Math Financ 9(3):203-228

Atherton E, French N, Gabrielli L (2008) Decision theory and real estate development: a note on uncertainty. J Eur Real Estate Res 1(2):162-182

Baginski SP, Conrad EJ, Hassel JM (1993) The effects of management forecast precision on equity pricing and on the assessment of earnings uncertainty. Account Rev 68(4):913-927

Bagozzi RP (2007) The legacy of the technology acceptance model and a proposal for a paradigm shift. J Assoc Inf Syst 8(4):244-254

Balzer WK, Hammer LB, Sumner KE, Birchenough TR, Parham Martens S, Raymark PH (1994) Effects of cognitive feedback components, display format, and elabortation on performance. Organ Behav Hum Decis Process 58(3):369-385

Barr SH, Sharda R (1997) Effectiveness of decision support systems: development or reliance effect? Decis Support Syst 21(2):133-146

Bellman L, Öhman P (2016) Authorised property appraisers perceptions of commercial property valuation. J Prop Invest Financ 34(3):225-248

Belsky E, Can A, Megbolugbe IF (1998) A primer on geographic information systems in mortgage finance. J Hous Res 9(1):5-31

Ben-Shahar D, Golan R (2014) Real estate and personality. J Behav Exp Econ 53:111-119

Ben-Zvi T (2009) Measuring DSS effectiveness in a simulated environment. Proc Fifteenth Am Conf Inf Syst 2009(675):1-12

Bhandari G, Hassanein K, Deaves R (2008) Debiasing investors with decision support systems: an experimental investigation. Decis Support Syst 46(1):399-410

Black R (1997) Expert property negotiators and pricing information, revisited. J Prop Valuat Invest 15(3):274-281

Black RT, Diaz J III (1996) The use of information versus asking price in the real property negotiation process. J Prop Res 13(4):287-297

Bokhari S, Geltner D (2011) Loss aversion and anchoring in commercial real estate pricing: empirical evidence and price index implications. Real Estate Econ 39(4):635-670

Bolger F, Önkal-Atay D (2004) The effects of feedback on judgmental interval predictions. Int J Forecast 20(1):29-39

de Bondt W, Thaler R (1985) Does the stock market overreact? J Finance 40(3):793-805

Brewer NT, Chapman GB (2002) The fragile basic anchoring effect. J Behav Decis Making 15(1):65-77

Brown LD (1996) Analyst forecasting errors and their implications for security analysis: an alternative perspective. Financ Anal J 52(1):40-47

Brown LD (1997) Analyst forecasting errors: additional evidence. Financial Anal J 53(6):81-88

de Bruin A, Flint-Hartle S (2003) A bounded rationality framework for property investment behaviour. J Prop Invest Finance 21(3):271-284

Brunswik E (1955) Representative design and probabilistic theory in a functional psychology. Psychol Rev 62(3):193-217 
Bucchianeri GW, Minson JA (2013) A homeowner's dilemma: anchoring in residential real estate transactions. J Econ Behav Organ 89:76-92

Byrne P, Jackson C, Lee S (2013) Bias or rationality? The case of UK commercial real estate investment. J Eur Real Estate Res 6(1):6-33

Carter CR, Kaufmann L, Michel A (2007) Behavioral supply management: a taxonomy of judgment and decision-making biases. Int J Phys Distrib Logist Manag 37(8):631-669

Cebi S, Kahraman C (2010) Fuzzy multicriteria group decision making for real estate investments. Proc Inst Mech Eng Part I J Syst Control Eng 224(4):457-470

Chen C-W, Koufaris M (2015) The impact of decision support system features on user overconfidence and risky behavior. Eur J Inf Syst 24(6):607-623

Cho M, Megbolugbe IF (1996) An empirical analysis of property appraisal and mortgage redlining. J Real Estate Finance Econ 13(1):45-55

Cohen MD, March JG, Olsen JP (1972) A garbage can model of organizational choice. Adm Sci Q 17(1):1-25

Coutinho-Rodrigues J, Simão A, Antunes CH (2011) A GIS-based multicriteria spatial decision support system for planning urban infrastructures. Decis Support Syst 51(3):720-726

Cypher M, Hansz JA (2003) Does assessed value influence market value judgments? J Prop Res 20(4):305-318

Czernkowski R (1990) Expert systems in real estate valuation. J Valuat 8(4):376-393

Das TK, Teng B-S (1999) Cognitive biases and strategic decision processes: an integrative perspective. J Management Studies 36(6):757-778

Dawes RM, Faust D, Meehl PE (1989) Clinical versus actuarial judgment. Science 243(31):1668-1673

Diaz J III (1990) How appraisers do their work: a test of the appraisal process and the development of a descriptive model. J Real Estate Res 5(1):1-15

Diaz J III (1997) An investigation into the impact of previous expert value estimates on appraisal judgment. J Real Estate Res 13(1):57-66

Diaz J III (1999) The first decade of behavioral research in the discipline of property. J Prop Invest Finance 17(4):326-332

Diaz J III, Hansz JA (2007) Understanding the behavioural paradigm in property research. Pac Rim Prop Res J 13(1):16-34

Diaz J III, Gallimore P, Levy D (2004) Multicultural examination of valuation behaviour. J Prop Invest Finance 22(4):339-346

Diaz J III, Zhao R, Black R (1999) Does contingent reward reduce negotiation anchoring? J Prop Invest Finance 17(4):374-379

Diekmann KA, Tenbrunsel AE, Shah PP, Schroth HA, Bazerman MH (1996) The descriptive and prescriptive use of previous purchase price in negotiations. Organ Behav Hum Decis Process 66(2):179-191

Ellis C, Wilson P (2006) Expert system portfolios of Australian and UK Securitised property investments. Pac Rim Prop Res J 12(1):107-127

Evans K, Lausberg C, Sui Sang How JJ (2019) Reducing property appraisal bias with decision support systems: an experimental investigation in the south African property market. J Afr Real Estate Res 4(1):108-138

Farragher EJ, Savage A (2008) An investigation of real estate investment decision-making practices. J Real Estate Pract Educ 11(1):29-40

Forgionne GA (1996) Forecasting army housing supply with a DSS-delivered econometric model. Omega 24(5):561-576

French N (2001) Decision theory and real estate investment: an analysis of the decision-making process of real estate fund managers. Manag Decis Econ 22(7):399-410

French N, French S (1997) Decision theory and real estate investment. J Prop Valuat Invest 15(3):226-232

Gallimore P (1994) Aspects of information processing in valuation judgement and choice. J Prop Res 11(2):97-110

Gallimore P (1996) Confirmation bias in the valuation process: a test for corroborating evidence. J Prop Res 13(4):261-273

Gallimore P, McAllister P (2004) Expert judgement in the processes of commercial property market forecasting. J Prop Res 21(4):337-360

Gallimore P, Wolverton M (1997) Price-knowledge-induced bias: a cross-cultural comparison. J Prop Valuat Invest 15(3):261-273

Gallimore P, Hansz JA, Gray A (2000) Decision making in small property companies. J Prop Invest Financ 18(6):602-612 
Genesove D, Mayer C (2001) Loss aversion and seller behavior: evidence from the housing market. Q J Econ 116(4):1233-1260

George JF, Duffy K, Ahuja M (2000) Countering the anchoring and adjustment bias with decision support systems. Decis Support Syst 29(2):195-206

Gigerenzer G, Hoffrage U (1995) How to improve Bayesian reasoning without instruction: fequency formats. Psychol Rev 102(4):684-704

Gigerenzer G, Kurzenhäuser S (2005) Fast and frugal heuristics in medical decision making. In: Bibace R, Laird JD, Noller KL, Valsiner J (Hrsg) Science and medcine in dialogue, 1. Aufl. Praeger, Westport, S 3-15

Gonzalez AJ, Laureano-Ortiz R (1992) A case-based reasoning approach to real estate property appraisal. Expert Syst Appl 4(2):229-246

Goodwin P, Önkal-Atay D, Thomson ME, Pollock AC, Macaulay A (2004) Feedback-labelling synergies in judgmental stock price forecasting. Decis Support Syst 37(1):175-186

Greenhalgh T, Stones R, Swinglehurst D (2014) Choose and book: a sociological analysis of 'resistance' to an expert system. Soc Sci Med 104:210-219

Greer TH, Murtaza MB (2003) Technologies to improve the decision-making process of real estate appraisers: XML, intelligent agents, Avms, and web services. J Bus Econ Res 1(6):63-72

Grove WM, Zald DH, Lebow BS, Snitz BE, Nelson C (2000) Clinical versus mechanical prediction: a meta-analysis". Psychol Assess 12(1):19-30

Guo KL (2008) A decision-making model for more effective decision making by health care managers. Health Care Manag 27(2):118-127

Han S-Y, Kim TJ (1990) ESSAS: expert system for site analysis and selection. In: Kim TJ, Wiggins LL, Wright JR (Hrsg) Expert systems: applications to urban planning. Springer, New York, S 145-158

Han S-Y, Kim TJ, Adiguzel I (1991) Xplanner: a knowledge-based decision support system for facility management and planning. Environ Plan B Plan Des 18(2):205-224

Hansz JA, Diaz J III (2001) Valuation bias in commercial appraisal: a transaction price feedback experiment. Real Estate Econ 29(4):553-565

Harvey N, Koehler DJ, Ayton P (1997) Judgments of decision effectiveness. Actor-observer differences in overconfidence. Organ Behav Hum Decis Process 70(3):267-282

Havard T (1999) Do valuers have a greater tendency to adjust a previous valuation upwards or downwards? J Prop Invest Financ 17(4):365-373

Heinen E (1969) Zum Wissenschaftsprogramm der entscheidungsorientierten Betriebswirtschaftslehre. Z Betriebswirtsch 39(4):207-220

Herzog SM, Hertwig R (2009) The wisdom of many in one mind. Psychol Sci 20(2):231-237

Hodgkinson GP, Bown NJ, Maule AJ, Glaister KW, Pearman AD (1999) Breaking the frame: an analysis of strategic cognition and decision making under uncertainty. Strat Mgmt J 20(10):977-985

Hoffman JJ, Schniederjans MJ, Sirmans GS (1990) A multi-criteria model for corporate property evaluation. J Real Estate Res 5(3):285-300

Hornig S (1993) Reading risk: public response to print media accounts of technological risk. Public Underst Sci 2(2):95-109

HosseinKefi A, Kazemipoor H, AfsharKazem MA (2013) Design and implementation of fuzzy expert system for real estate recommendation. Int J Inf Secur Syst Manag 2(1):142-147

Huber GP (1981) The nature of organizational decision making and the design of decision support systems. Mis Q 5(2):1-10

Introne J, Iandoli L (2014) Improving decision-making performance through argumentation: an argumentbased decision support system to compute with evidence. Decis Support Syst 64:79-89

Jackson C, Orr A (2011) Real estate stock selection and attribute preferences. J Prop Res 28(4):317-339

Johnson WB (1982) The impact of confidence interval information on probability judgements. Account Organ Soc 7(4):349-367

Kahneman D (2003) A perspective on judgment and choice: mapping bounded rationality. Am Psychol 58(9):697-720

Kahneman D, Tversky A (1979) Prospect theory: an analysis of decision under risk. Econometrica 47(2):263-292

Kaklauskas A, Gikys M (2005) Increasing efficiency of multiple listing service systems applying webbased decision support system for real estate. J Civ Eng Manag 11(2):91-97

Kaklauskas A, Zavadskas EK, Banaitis A, Satkauskas G (2007) Defining the utility and market value of real estate: a multiple criteria approach. Int J Strateg Prop Manag 11(2):107-120

Kaklauskas A, Zavadskas EK, Raslanas S (2009) Modelling of real estate sector: the case for lithuania. Transform Bus Econ 8(1):101-120 
Kaklauskas A, Zavadskas EK, Vainiunas P (2001) Efficiency increase of real estate E-business systems by applying multiple criteria decision support systems. $8^{\text {th }}$ European Real Estate Society ConferenceERES Alicante, S 1-7

Karelaia N, Hogarth RM (2008) Determinants of linear judgment: a meta-analysis of lens model studies. Psychol Bull 134(3):404-426

Kasper GM (1996) A theory of decision support system design for user calibration. Inf Syst Res $7(2): 215-232$

Kayande U, de Bruyn A, Lilien GL, Rangaswamy A, van Bruggen GH (2009) How incorporating feedback mechanisms in a DSS affects DSS evaluations. Inf Syst Res 20(4):527-546

Kettani O, Khelifi K (2001) PariTOP: a goal programming-based software for real estate assessment. Eur J Oper Res 133(2):362-376

Kettani O, Oral M (2015) Designing and implementing a real estate appraisal system: the case of Québec province, Canada. Socio Econ Plann Sci 49:1-9

Kilpatrick J (2011) Expert systems and mass appraisal. J Prop Invest Financ 29(4/5):529-550

Kisilevich S, Keim D, Rokach L (2013) A GIS-based decision support system for hotel room rate estimation and temporal price prediction: the hotel brokers' context. Decis Support Syst 54(2):1119-1133

Klein G (1998) Sources of power: how people make decisions, 1. Aufl. MIT Press, Cambridge

Lagnado DA, Newell BR, Kahan S, Shanks DR (2006) Insight and strategy in multiple-cue learning. J Exp Psychol Gen 135(2):162-183

Larranz B (2011) An expert system for online residential properties valuation. Rev Econ Financ 1(2):69-82

Lausberg C, Dust A (2017) Reducing the appraisal bias in manual valuations with decision support systems. In: d'Amato M, Kauko T (Hrsg) Advances in automated valuation modeling: aVM after the non-agency mortgage crisis, studies in systems, decision and control. Springer, Cham, S 331-343

Lawrence M, O'Connor M (1996) Judgement or models: the importance of task differences. Omega 24(3):245-254

Lawrence M, Goodwin P, O’Connor M, Önkal D (2006) Judgmental forecasting: a review of progress over the last 25 years. Int J Forecast 22(3):493-518

Leelarasamee Y (2005) A decision support system for income-producing real estate development feasibility analysis and alternative assessment: Diss. A\&M University, Texas

Li H, Yu L, Cheng E (2005) A GIS-based site selection system for real estate projects. Constr Innov 5(4):231-241

Lifka S (2009) Entscheidungsanalysen in der Immobilienwirtschaft. Wirtschaft \& Raum, Bd. 18. Utz, München (Zugl.: München, Univ., Diss)

Lin T-C, Chang H (2012) How do appraisers absorb market information in property valuation? Prop Manag 30(2):190-206

Lindblom CE (1959) The science of muddling through. Public Adm Rev 19(2):79-88

Ling DC, Naranjo A, Petrova MT (2016) Search costs, behavioral biases, and information intermediary effects. J Real Estate Financ Econ 35(9):1-38

Lord CG, Lepper MR, Preston E (1984) Considering the opposite: a corrective strategy for social judgment. J Pers Soc Psychol 47(6):1231-1243

Lowe SP, Stanard JN (1997) An integrated dynamic financial analysis and decision support system for a property catastrophe reinsurer. ASTIN Bull 27(2):339-371

Lowies GA, Hall JH, Cloete CE (2016) Heuristic-driven bias in property investment decision-making in South Africa. J Prop Invest Financ 34(1):51-67

Luhmann N (1997) Die Moral des Risikos und das Risiko der Moral. In: Bechmann G (Hrsg) Risiko und Gesellschaft: Grundlagen und Ergebnisse interdisziplinärer Risikoforschung, 2. Aufl. Westdeutscher Verlag, Opladen, S 327-338

MacCowan RJ, Orr AM (2008) A behavioural study of the decision processes underpinning disposals by property fund managers. J Prop Invest Financ 26(4):342-361

Mahlendorf MD (2008) Verhaltensorientiertes Controlling in der Praxis - eine am Beispiel verspäteter Projektabbrüche illustrierte Systematik zur Identifikation, Bewertung und Auswahl von Controllingmaßnahmen. Control Manag Rev 52(1):104-112

Markowitz H (1952) Portfolio selection. J Finance 7(1):77-91

Marshall A (1920) Principles of economics, 8. Aufl. Macmillan \& Co, London

Mathews BP, Diamantopoulos A (1986) Managerial intervention in forecasting. An empirical investigation of forecast manipulation. Int J Res Mark 3(1):3-10

Mathews BP, Diamantopoulos A (1989) Factors affecting subjective revision in forecasting. Int J Res Mark 6(4):283-297 
McCluskey W, Anand S (1999) The application of intelligent hybrid techniques for the mass appraisal of residential properties. J Prop Invest Financ 17(3):218-239

Meehl PE (1954) Clinical versus statistical prediction: a theoretical analysis and a review of the evidence, 1. Aufl. Echo Point Books \& Media, Guilford (reprint 2013)

Meehl PE (1965) Seer over sign: the first good example. J Exp Res Pers 1(1):27-32

Metzner S (2013) Immobilienökonomische Methoden zur Entscheidungsunterstützung und Planung: Selektion und Integration im Rahmen der wertorientierten Steuerung von Immobilienportfolios institutioneller Anleger. MV-Wissenschaft, Münster

Meyer K, Pfnür A (2015) Kognitiv verzerrte Entscheidungen als Ursache für Ineffizienzen in der Immobilienprojektentwicklung: Managementorientierte Zusammenfassung der Ergebnisse einer empirischen Studie, Arbeitspapiere zur immobilienwirtschaftlichen Forschung und Praxis. Working Paper, Bd. 30. Techn. Univ. Darmstadt, Darmstadt

Mitchell DJ, Russo JE, Pennington N (1989) Back to the future: temporal perspective in the explanation of events. J Behav Decis Making 2(1):25-38

Montazemi AR, Wang F, Nainar SMK, Bart CK (1996) On the effectiveness of decisional guidance. Decis Support Syst 18(2):181-198

Moore JS (1992) A prototype expert decision support system for the market appraisal of the single family residence. Decis Sci 23(6):1408-1422

Musa AG, Daramola O, Owoloko A, Olugbara O (2013) A neural-CBR system for real property valuation. J Emerg Trends Comput Inf Sci 4(8):611-622

Natividade-Jesus E, Coutinho-Rodrigues J, Antunes CH (2007) A multicriteria decision support system for housing evaluation. Decis Support Syst 43(3):779-790

von Neumann J, Morgenstern O (1947) Theory of games and economic behavior, 2. Aufl. Princeton University Press, Princeton

de Neys W (2006) Dual processing in reasoning: two systems but one reasoner. Psychol Sci 17(5):428-433

Northcraft GB, Neale MA (1987) Experts, amateurs, and real estate: an anchoring-and-adjustment perspective on property pricing decisions. Organ Behav Hum Decis Process 39(1):84-97

Öhman P, Söderberg B, Westerdahl S (2013) Property investor behaviour: qualitative analysis of a very large transaction. J Prop Invest Financ 31(6):522-544

Parker D (2014) Property investment decision making by Australian REITs. J Prop Invest Finance 32(5):456-473

Pedersen CS, Satchell SE (1998) An extended family of financial-risk measures. Geneva Pap Risk Insur Theory 23(2):89-117

Peterson K (1993) Spatial decision support systems for real estate investment analysis. Int J Geogr Inf Syst 7(4):379-392

Peterson K (1998) Development of spatial decision support systems for residential real estate. J Hous Res 9(1):135-156

Pfeffer J (1981) Power in organizations, 1. Aufl. Pitman Publishing, Marshfield

Phillips S, Martin J, Dainty A, Price A (2007) Uncertainty in best value decision making. J Financial Manag Prop Constr 12(2):63-72

Podor A, Nyiri J (2010) GIS application in real estate investment. Sci J Riga Tech Univ 20:94-99

Pommer A (2007) Entscheidungsunterstützung in der Immobilienprojektentwicklung, 1. Aufl. Schriftenreihe Bau- und Immobilienmanagement, Bd. 6. VDG, Weimar, Kromsdorf

Power DJ, Burstein F, Sharda R (2011) Reflections on the past and future of decision support systems: perspective of eleven pioneers. In: Schuff D, Paradice D, Burstein F, Power DJ, Sharda R (Hrsg) Decision support: an examination of the DSS discipline, annals of information systems. Springer, New York, S 25-48

Pownall G, Wasley C, Waymire G (1993) The stock price effects of alternative types of management earnings forecasts. Account Rev 68(4):896-912

Ratcliffe J (2001) Imagineering global real estate: a property foresight exercise. Foresight 3(5):453-465

Reddy W, Higgins D, Wakefield R (2014) An investigation of property-related decision practice of Australian fund managers. J Prop Invest Finance 32(3):282-305

van Reedt Dortland M, Voordijk H, Dewulf G (2012) Towards a decision support tool for real estate management in the health sector using real options and scenario planning. J Corp Real Estate 14(3):140-156

Remus W, O’Connor M, Griggs K (1996) Does feedback improve the accuracy of recurrent judgmental forecasts? Organ Behav Hum Decis Process 66(1):22-30

Roberts C, Henneberry J (2007) Exploring office investment decision-making in different European contexts. J Prop Invest Finance 25(3):289-305 
Rockafellar RT, Uryasev S, Zabarankin M (2006) Generalized deviations in risk analysis. Finance Stochastics 10(1):51-74

Rossini P (2000) Using expert systems and artificial intelligence for real estate forecasting. Sixth Annual Pacific-Rim Real Estate Society Conference. Working paper, S 1-10

Saaty RW (1987) The analytic hierarchy process-what it is and how it is used. Math Model 9(3-5): $161-176$

Sah V (2011) Asset acquisition criteria: a process tracing investigation into real estate investment decision making. J Prop Invest Financ 29(1):7-18

Sainfort FC, Gustafson DH, Bosworth K, Hawkins RP (1990) Decision support systems effectiveness: conceptual framework and empirical evaluation. Organ Behav Hum Decis Process 45(2):232-252

Savage LJ (1972) The Foundations of Statistics, 2. Aufl. Dover Publications, New York

Schwarz N, Bless H, Strack F, Klumpp G, Rittenauer-Schatka H, Simons A (1991) Ease of retrieval as information: another look at the availability heuristic. J Pers Soc Psychol 61(2):195-202

Seiler MJ, Seiler VL (2010) Mitigating investor risk-seeking behavior in a down real estate market. J Behav Financ 11(3):161-167

Seiler MJ, Seiler VL, Lane MA (2012) Mental accounting and false reference points in real estate investment decision making. J Behav Financ 13(1):17-26

Seiler MJ, Seiler VL, Traub S, Harrison DM (2008) Regret aversion and false reference points in residential real estate. J Real Estate Res 30(4):461-474

Sengupta K (1995) Cognitive feedback in environments characterized by irrelevant information. Omega 23(2):125-143

Sharda R, Barr SH, McDonell JC (1988) Decision support systems effectiveness: a review and an empirical test. Manage Sci 34(2):139-159

Shefrin H (2016) Behavioral risk management: managing the psychology that drives decisions and influences operational risk, 1. Aufl. Palgrave Macmillan, New York

Simon H (1955) A behavioral model of rational choice. Q J Econ 69(1):99-118

Simon H (1977) The new science of management decision. Englewood Cliffs, Prentice-Hall

Simoni MC (2011) A decision model for real estate portfolio valuation and Optimisation: under consideration of real estate physical characteristics: Diss, 1. Aufl. HWZ Schriftenreihe für Betriebs- und Bildungsökonomie, Bd. 12. Haupt, Bern

Sloman SA (1996) The empirical case for two systems of reasoning”. Psychol Bull 119(1):3-22

Slovic P (1999) Trust emotion sex politics and science: surveying the risk-assessment battlefield. Risk Anal 19(4):689-701

Soll JB, Klayman J (2004) Overconfidence in interval estimates. J Exp Psychol Learn Mem Cogn 30(2):299

Stanovich KE, West RF (2000) Individual differences in reasoning: implications for the rationality debate? Behav Brain Sci 23(5):645-726

Strack F, Mussweiler T (1997) Explaining the enigmatic anchoring effect: mechanisms of selective accessibility. J Pers Soc Psychol 73(3):437-446

Taillandier F, Abi-Zeid I, Taillandier P, Sauce G, Bonetto R (2014) An interactive decision support method for real estate management in a multi-criteria framework-REMIND. Int J Strateg Prop Manag 18(3):265-278

Tidwell OA, Gallimore P (2012) The influence of a decision support tool on real estate valuations. J Prop Res 31(1):45-63

Todd P, Benbasat I (1992) The use of information in decision making - an experimental investigation of the impact of computer-based decision aids. Mis Q 16(3):373-393

Trippi RR (1989) A decision support system for real estate investment portfolio management. Inf Manag 16(1):47-54

Trippi RR (1990) Decision support and expert systems for real estate investment decisions: a review. Interfaces 20(5):50-60

Tversky A, Kahneman D (1973) Availability: a heuristic for judging frequency and probability. Cogn Psychol 5(2):207-232

Tversky A, Kahneman D (1974) Judgment under uncertainty heuristics and biases. Science 185(4157): $1124-1131$

Udo G (1992) Rethinking the effectiveness measures of decision support systems. Inf Manag 22(2): 123-135

Urbanavičiene V, Kaklauskas A, Zavadskas EK (2009a) The conceptual model of construction and real estate negotiation. Int J Strateg Prop Manag 13(1):53-70 
Urbanavičiene V, Kaklauskas A, Zavadskas EK, Seniut M (2009b) The web-based real estate multiple criteria negotiation decision support system: a new generation of decision support systems. Int $\mathrm{J}$ Strateg Prop Manag 13(3):267-286

Valverde R (2010) An adaptive decision support station for real estate portfolio management. J Theor Appl Inf Technol 12(2):84-86

Valverde R (2011) A risk management decision support system for the real estate industry. Int J Inf Commun Technol Res 1(3):139-147

Venkatesh V, Bala H (2008) Technology acceptance model 3 and a research agenda on interventions. Decis Sci 39(2):273-315

Viezer TW (2010) The application of modern portfolio theory to real estate: a brief survey. In: Guerard JB (Hrsg) Handbook of portfolio construction-contemporary applications of Markotwitz techniques, 1. Aufl. Springer, Heidelberg, S 733-760

VÖB-Kommission für Bewertungsfragen (2006) VÖB-ImmobilienAnalyse: Instrument zur Beurteilung des Chance-/Risikoprofils von Immobilien, 1. Aufl. VÖB-Kommission für Bewertungsfragen, Berlin

Wang WK (2005) A knowledge-based decision support system for measuring the performance of government real estate investment. Expert Syst Appl 29(4):901-912

Wang K, Yuqing Z, Chan SH, Chau K (2000) Over-confidence and cycles in real estate markets: cases in Hong Kong and Asia. Int Real Estate Rev 3(1):93-108

Webby R, O'Connor M (1994) The effectiveness of decision support systems: the implications of task complexity and DSS sophistication. J Inf Technol 9(1):19-28

Wixom BH, Todd PA (2005) A theoretical integration of user satisfaction and technology acceptance. Inf Syst Res 16(1):85-102

Wofford L, Troilo M, Dorchester A (2011) Real estate valuation, cognitive risk, and translational research. J Prop Invest Financ 29(4/5):372-383

Ye X, Dent PR (2009) Invariance assumptions among the UK property professionals' decision making. J Eur Real Estate Res 2(1):56-68

York KM, Doherty ME, Kamouri J (1987) The influence of cue unreliability on judgment in a multiple cue probability learning task. Organ Behav Hum Decis Process 39(3):303-317

Zavadskas EK, Kaklauskas A (2009) Web-based intelligent decision support system for real estate. Intellect Econ 2(6):51-60

Zeng TQ, Zhou Q (2001) Optimal spatial decision making using GIS: a prototype of a real estate geographical information system (REGIS). Int J Geogr Inf Sci 15(4):307-321 